\title{
A CLASSIFICATION OF EQUIVARIANT GERBE CONNECTIONS
}

\author{
BYUNGDO PARK AND CORBETT REDDEN
}

\begin{abstract}
Let $G$ be a compact Lie group acting on a smooth manifold $M$. In this paper, we consider Meinrenken's $G$-equivariant bundle gerbe connections on $M$ as objects in a 2-groupoid. We prove this 2-category is equivalent to the 2-groupoid of gerbe connections on the differential quotient stack associated to $M$, and isomorphism classes of $G$-equivariant gerbe connections are classified by degree three differential equivariant cohomology. Finally, we consider the existence and uniqueness of conjugation-equivariant gerbe connections on compact semisimple Lie groups.
\end{abstract}

\section{Contents}

1. Introduction

2. Background and conventions

2.1. Sheaves of $\infty$-groupoids on the site of manifolds

2.2. Relevant examples

2.3. Cohomology and differential cohomology

3. The 2-groupoid of equivariant bundle gerbe connections 9

3.1. The definition 9

3.2. Basic properties 11

3.3. Fiber product morphisms 12

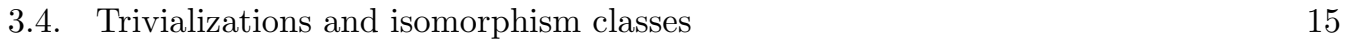

$\begin{array}{ll}3.5 . & \text { The induced simplicial gerbe } \\ & 18\end{array}$

4. Gerbes on the quotient stack 18

4.1. The functor between the two models 20

4.2. Proof of equivalence 24

5. Applications 26

5.1. Equivariant holonomy 27

5.2. Equivariant gerbes over compact semisimple Lie groups 28

$\begin{array}{lr}\text { 5.3. Higher gerbes } & 29\end{array}$

$\begin{array}{ll}\text { References } & 29\end{array}$

\section{INTRODUCTION}

While there exist several models for $S^{1}$-banded gerbes with connection, working directly with any of them necessarily involves technical details and subtleties, and the complexity grows rapidly should one consider $p$-gerbes for $p>1$. However, there is a relatively simple classification of gerbe connections up to isomorphism, which is given by degree 3 differential cohomology (Deligne cohomology) and fits into the pattern

$$
\widehat{H}^{1}(M) \cong C^{\infty}\left(M, S^{1}\right), \quad \widehat{H}^{2}(M) \cong \operatorname{Bun}_{S^{1}, \nabla}(M)_{/ \cong}, \quad \widehat{H}^{3}(M) \cong \operatorname{Grb}_{\nabla}(M) / \cong .
$$


Because the groups $\widehat{H}^{n}(M)$ fit into short exact sequences involving differential forms and ordinary cohomology, one can use elementary techniques to get a strong foothold in the gerbe world.

Assume now that $G$ is a compact Lie group acting smoothly on a finite-dimensional manifold $M$. This paper's aim is to further develop the theory of $G$-equivariant gerbe connections in a manner analogous to $G$-Bun $S_{S^{1}, \nabla}(M)$, the groupoid of $G$-equivariant $S^{1}$ bundles on $M$ with $G$-invariant connection. We do so by considering two specific models. The first is denoted $G-G_{r b}(M)$, the 2-groupoid of $G$-equivariant bundle gerbes on $M$ with $G$-equivariant connection. Its objects, which were defined by Meinrenken [Mei] and further investigated by Stiénon [Sti] and $\mathrm{Tu}-\mathrm{Xu}[\mathrm{TX}]$, are differential-geometric in the same way as Murray's bundle gerbes with connection [Mur]. We define a 2-categorical structure on $G$-Grb ${ }_{\nabla}(M)$ by adapting Waldorf's 2-groupoid structure on $\operatorname{Grb}_{\nabla}(M)$ in a straightforward way [Wal1].

The second model uses the language of higher stacks, or sheaves of $\infty$-groupoids on the site of manifolds. For $M \in G$-Man we consider the differential quotient stack $\mathcal{E}_{\nabla} G \times{ }_{G} M$, which associates to every test manifold $X$ the groupoid of principal $G$-bundles with connection $(P, \Theta) \in \operatorname{Bun}_{G, \nabla}(X)$, together with $G$-equivariant map $f: P \rightarrow M$. In [Red1], the differential equivariant cohomology groups were defined as

$$
\widehat{H}_{G}^{n}(M):=\widehat{H}^{n}\left(\mathcal{E}_{\nabla} G \times_{G} M\right),
$$

suitably interpreted, and it was shown that they fit into short exact sequences (2.21)(2.23) involving the Borel equivariant cohomology $H_{G}^{*}(M ;-):=H^{*}\left(E G \times_{G} M ;-\right)$ and the equivariant differential forms $\Omega_{G}^{*}(M)$ of Cartan-Weil. Furthermore, there are isomorphisms generalizing (1.1) in degrees one and two:

$$
\widehat{H}_{G}^{1}(M) \cong C^{\infty}\left(M ; S^{1}\right)^{G}, \quad \widehat{H}_{G}^{2}(M) \cong G-\operatorname{Bun}_{S^{1}, \nabla}(M) / \cong .
$$

It was further shown in [Red2] that there is a natural equivalence of groupoids

$$
G-\operatorname{Bun}_{K, \nabla}(M) \stackrel{\cong}{\leftrightarrows} \operatorname{Bun}_{K, \nabla}\left(\mathcal{E}_{\nabla} G \times{ }_{G} M\right)
$$

for any Lie groups $G, K$. When combined with the properties of $\widehat{H}_{G}^{3}(M)$, this strongly suggests that $\operatorname{Grb}_{\nabla}\left(\mathcal{E}_{\nabla} G \times_{G} M\right)$ should be a valid model for $G$-equivariant gerbe connections.

That hope is confirmed by the two primary results of this paper, Theorems 4.15 and 4.16. The first theorem states

$$
G-\operatorname{Grb}_{\nabla}(M)_{/ \cong} \stackrel{\cong}{\longrightarrow} \widehat{H}_{G}^{3}(M),
$$

that isomorphism classes of $G$-equivariant bundle gerbe connections are classified by degree 3 differential equivariant cohomology. The second theorem proves that a natural functor

$$
G-\operatorname{Grb}_{\nabla}(M) \stackrel{\simeq}{\longrightarrow} \operatorname{Grb}_{\nabla}\left(\mathcal{E}_{\nabla} G \times_{G} M\right)
$$

is an equivalence of 2-groupoids. As explained in works such as [NS], a natural approach to equivariance in higher geometry is to use $\mathcal{E} G \times_{G} M$ and the language of sheaves. The essential point of this paper is that when connections are involved, one should consider $\mathcal{E}_{\nabla} G \times_{G} M$ instead of $\mathcal{E} G \times_{G} M$.

As a sample application of Theorem 4.15, we consider the case where $G$ is a compact semisimple Lie group acting on itself by conjugation. Theorem 5.4 gives a simple proof of the existence and uniqueness of certain $G$-equivariant gerbe connections on $G$. Such gerbes have been considered in several other works, including [BXZ, Bry2, GR, Mei, MW]. 
The paper is structured in the following way. Section 2 contains a brief exposition of existing knowledge that will be utilized in the paper. The inclusion of this section serves the twofold purpose of establishing notations and conventions, and making the paper as selfcontained as possible. Section 3 contains an explicit definition of the 2-groupoid $G$-Grb $\nabla(M)$ and proves many basic properties, most of which follow easily from existing work in [Wal1, Mei, Sti]. Section 4 introduces the second model of $\operatorname{Grb}_{\nabla}\left(\mathcal{E}_{\nabla} G \times_{G} M\right)$, and we use the Weil homomorphism to define the functor (1.3). Making use of a result by $\mathrm{Tu}-\mathrm{Xu}$ [TX], we are able to prove that (1.3) is a bijection on isomorphism classes, and we use the main result from [Red2] to prove that it induces equivalences of automorphism groupoids. Finally, Section 5 contains a brief discussion of equivariant holonomy, and it proves a unique existence result for Ad-equivariant gerbes on compact semisimple Lie groups. We also explain a natural model for equivariant connections on $p$-gerbes for $p>1$.

Finally, it is worth noting that gerbes, both with and without connection, have played an important role in mathematical and physical problems, including twisted/differential $K$-theory and Ramond-Ramond field classifications $\left[\mathrm{BCM}^{+}, \mathrm{KV}\right.$, Par, GL], Wess-ZuminoWitten models [SSW], string structures [Wal2], and even topological insulators [Gaw, MT]. In each of these situations, it is natural to seek equivariant generalizations. The tools developed in this paper should aid especially in answering existence/uniqueness questions and in better developing functorial constructions. In particular, the formal properties of $\operatorname{Grb}_{\nabla}\left(\mathcal{E}_{\nabla} G \times_{G} M\right)$, displayed in (4.1), make equivariant gerbe connections excellent candidates for WZW terms in gauged sigma models [Wit, FOS, GSW1, GSW2, BE].

Acknowledgements. B.P. thanks Hausdorff Research Institute for Mathematics and Max Planck Institute for Mathematics for their support and hospitality during his visits. The authors thank Jim Stasheff for detailed comments on a previous draft.

\section{BACKGROUND AND CONVENTIONS}

We begin by recalling background information and explaining notation, most of which follows the conventions from [Red1, Red2, FH]; see [BNV, Lur] for further details. Throughout this paper, we work in the large category Man of smooth manifolds with smooth maps.

2.1. Sheaves of $\infty$-groupoids on the site of manifolds. While we use the language of sheaves and infinity categories, the reader unfamiliar with such terms should not worry. Our sheaves are simply structures that naturally pullback, and our infinity-groupoids arise from ordinary $n$-categorical structures with $n \leq 2$. The particular model of $\infty$-Gpd we use is the category of Kan complexes, viewed as a simplicially enriched full subcategory of simplicial sets. Most of our arguments, though, are model-independent.

Any set is naturally a groupoid (a category whose morphisms are all invertible) with only the identity morphisms, and any groupoid determines a 2-groupoid with trivially defined 2-morphisms. We freely use these natural embeddings (fully faithful functors)

$$
\text { Set } \hookrightarrow \text { Gpd } \hookrightarrow 2-G p d \hookrightarrow \infty-G p d,
$$

but we do not denote them by extra symbols. For example, the map Gpd $\hookrightarrow \infty$-Gpd is the nerve construction, but we usually avoid the symbol $N$ and instead write Gpd $\subset \infty$-Gpd. While this may be technically imprecise, we believe it is easier to read and will cause no confusion.

Remark 2.1. Many of our constructions can be regarded as strict 2-categories. However, the main results come after passing to $\infty$-Gpd, so issues such as strict 2-category versus 
bicategory will be irrelevant. Therefore, we use the term 2 -groupoid to refer to $\infty$-groupoids whose homotopy groups vanish above degree 2 .

A morphism $\varphi: \mathcal{C} \rightarrow \mathcal{C}^{\prime}$ in $\infty$-Gpd is an equivalence of $\infty$-groupoids if it is a homotopy equivalence between the two underlying simplicial sets. Since $\mathcal{C}, \mathcal{C}^{\prime}$ are Kan complexes, $\varphi$ is an equivalence if and only if it induces isomorphisms between all homotopy groups. When $\mathcal{C}, \mathcal{C}^{\prime}$ are 2-groupoids, the homotopy groups $\pi_{n}\left(\mathcal{C}^{\left({ }^{\prime}\right)}\right)$ vanish for all $n>2$. Hence $\varphi$ is an equivalence of 2-groupoids if and only if: $\varphi$ induces a bijection between isomorphism classes of objects $\pi_{0}(\mathcal{C}) \cong \pi_{0}\left(\mathcal{C}^{\prime}\right)$; and $\varphi$ induces an equivalence of categories $\operatorname{Aut}_{\mathcal{C}}(x) \simeq$ Aut $_{\mathcal{C}^{\prime}}(\varphi(x))$ for all objects $x \in \mathcal{C}$. Note that since all morphisms are invertible, this implies the equivalence of categories $\mathcal{C}(x, y) \simeq \mathcal{C}^{\prime}(\varphi(x), \varphi(y))$ for all $x, y \in \mathcal{C}$. While we usually use the symbol $\simeq$ to denote equivalences, we sometimes use $\cong$ when an explicit functor has been constructed.

Define Pre $_{\infty}$ as the $(\infty, 1)$-category of $\infty$-Gpd-valued presheaves on the site of manifolds. It is the collection of functors $\operatorname{Pre}_{\infty}:=\mathrm{Fun}\left(\mathrm{Man}^{\mathrm{op}}, \infty-\mathrm{Gpd}\right)$. We will be primarily interested in presheaves that satisfy a certain sheaf condition.

For $M \in$ Man, we call a surjective submersion $Y \stackrel{\pi}{\rightarrow} M$ a cover of $M$ and define the associated fiber products

$$
Y^{[k]}:=Y \times_{M} \cdots \times_{M} Y=\left\{\left(y_{1}, \ldots, y_{k}\right) \in Y^{k} \mid \pi\left(y_{1}\right)=\cdots=\pi\left(y_{k}\right)\right\},
$$

with associated projection maps $\pi_{i_{1} i_{2} \cdots i_{k}}: Y^{[p]} \rightarrow Y^{[k]}$. These manifolds form a simplicial manifold $Y^{[\bullet]}: \Delta^{\mathrm{op}} \rightarrow$ Man. When $Y \rightarrow M$ is $G$-equivariant, we refer to it as a $G$-cover.

Define $\operatorname{Shv}_{\infty}$ as the full subcategory of $\operatorname{Pre}_{\infty}$ whose objects $\mathcal{F}$ satisfy the following sheaf/descent condition: for any cover $U \rightarrow X$ with discrete fibers, the natural map

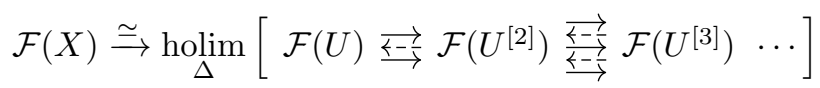

is an equivalence in $\infty$-Gpd. This recovers the usual sheaf condition when $\mathcal{F}$ is Set-valued, and it recovers the stack condition for presheaves of groupoids (for this reason objects in $S h v_{\infty}$ are often called $\infty$-stacks). The inclusion $\operatorname{Shv}_{\infty} \hookrightarrow \operatorname{Pre}_{\infty}$ has a left adjoint $\mathbb{L}$, which we call the sheafification, that gives natural equivalences of $\infty$-groupoids

$$
\operatorname{Pre}_{\infty}\left(\mathcal{F}, \mathcal{F}^{\prime}\right) \cong \operatorname{Shv}_{\infty}\left(\mathbb{L}(\mathcal{F}), \mathcal{F}^{\prime}\right)
$$

for all $\mathcal{F} \in \operatorname{Pre}_{\infty}, \mathcal{F}^{\prime} \in \operatorname{Shv}_{\infty}$. Here, $\operatorname{Pre}_{\infty}\left(\mathcal{F}, \mathcal{F}^{\prime}\right) \in \infty$-Gpd is the collection of maps $\mathcal{F} \rightarrow \mathcal{F}^{\prime}$, which are natural transformations between the functors $\mathcal{F}, \mathcal{F}^{\prime} \in \operatorname{Fun}\left(\operatorname{Man}^{\mathrm{op}}, \infty\right.$-Gpd).

\subsection{Relevant examples.}

Example 2.4 (Smooth maps and Yoneda). Any manifold $M \in$ Man defines a sheaf $M \in$ Shv $v_{\infty}$, whose value on a test manifold $X$ is given by the set of smooth functions

$$
M(X):=C^{\infty}(X, M) \in \operatorname{Set} \subset \infty \text {-Gpd. }
$$

The Yoneda Lemma states there is a natural equivalence $\operatorname{Pre}_{\infty}(M, \mathcal{F}) \cong \mathcal{F}(M) \in \infty$-Gpd for any $\mathcal{F} \in \operatorname{Pre}_{\infty}$.

Example 2.5 (Differential forms). Let $\Omega^{n} \in \mathrm{Shv}_{\infty}$ be the sheaf that assigns to any test manifold $X$ the differential $n$-forms $\Omega^{n}(X) \in$ Set $\subset \infty$-Gpd. Note that $\Omega^{n}(X)$ is considered as a category with no non-trivial morphisms, as opposed to the $\infty$-groupoid obtained from the de Rham complex $\cdots \rightarrow \Omega^{n-1}(X) \stackrel{d}{\rightarrow} \Omega^{n}(X)$. 
Example 2.6 (Bundles). For $X \in$ Man and $K$ a Lie group, let $\operatorname{Bun}_{K, \nabla}(X)$ denote the groupoid of principal $K$-bundles with connection on $X$; morphisms are bundle isomorphisms preserving the connection and covering the identity map on $X$. Principal bundles and connections naturally pullback along smooth maps, and they can be glued, thus defining a stack $\mathcal{B}_{\nabla} K \in \mathrm{Shv}_{\infty}$ by

$$
\left(\mathcal{B}_{\nabla} K\right)(X):=\operatorname{Bun}_{K, \nabla}(X) \in \mathrm{Gpd} \subset \infty-\mathrm{Gpd} .
$$

Similarly, we can ignore connections and define a stack by $(\mathcal{B} K)(X):=\operatorname{Bun}_{K}(X)$.

For $G$ a Lie group, assumed to be compact throughout this paper, let $G$-Man denote the category whose objects are smooth manifolds equipped with a smooth (left) $G$-action, and whose morphisms are $G$-equivariant smooth maps. We follow the convention that $M \in G$-Man has a left $G$-action, $P \in \operatorname{Bun}_{G}(X)$ has a right $G$-action, and inverses are used to switch between left/right actions when necessary.

Example 2.7 (Quotient stacks). If $M \in G$-Man, define the differential quotient stack $\mathcal{E}_{\nabla} G \times_{G} M \in \operatorname{Shv}_{\infty}$ by assigning to a test manifold $X$ the groupoid whose objects are $(P, \Theta, f)$, where $(P, \Theta) \in \operatorname{Bun}_{G, \nabla}(X)$ and $f: P \rightarrow M$ is a $G$-equivariant map. Morphisms are given by morphisms in $\operatorname{Bun}_{G, \nabla}(X)$ compatible with maps $f$. When $M=$ pt, which is trivially a $G$-manifold, then $\mathcal{E}_{\nabla} G \times_{G} \mathrm{pt}=\mathcal{B}_{\nabla} G$. The quotient stack $\mathcal{E} G \times_{G} M \in \mathrm{Shv}_{\infty}$ is defined similarly, but without including connections. The quotient stack serves as a replacement for the more familiar homotopy quotient $E G \times_{G} M$, and the adjective "differential" indicates the presence of local geometric data given by differential forms.

Remark 2.8. For $M \in G$-Man, the action groupoid is the Lie groupoid $(G \times M \rightrightarrows M)$, which becomes the simplicial manifold $M / / G=G \bullet \times M$ when viewed in $\infty-G p d$. The Yoneda embedding gives rise to a presheaf of groupoids with value

$$
(M / / G)(X):=N\left(C^{\infty}(X, G \times M) \rightrightarrows C^{\infty}(X, M)\right) \in \infty-\mathrm{Gpd}
$$

on a test manifold $X$. This presheaf does not satisfy the descent condition, but its sheafification is equivalent to the quotient stack $\mathbb{L}(M / / G) \stackrel{\simeq}{\rightarrow} \mathcal{E} G \times_{G} M \in \operatorname{Shv}_{\infty}$. Similarly, the differential quotient stack $\mathcal{E}_{\nabla} G \times_{G} M$ is equivalent to the sheafification of the differential action groupoid $M / / \nabla G:=N\left(G \times \Omega^{1}(\mathfrak{g}) \times M \rightrightarrows \Omega^{1}(\mathfrak{g}) \times M\right)$, where $\Omega^{1}(\mathfrak{g})$ is the sheaf of $\mathfrak{g}$-valued 1-forms $[\mathrm{FH}]$.

When $B K$ is regarded as a fixed topological space, there is no natural way to choose a classifying map $X \rightarrow B K$ for a given bundle with connection $(P, \Theta) \in \operatorname{Bun}_{K, \nabla}(X)$. In contrast, the Yoneda Lemma says that $(P, \Theta)$ is naturally equivalent to a map of stacks $X \stackrel{(P, \Theta)}{\longrightarrow} \mathcal{B}_{\nabla} K$. Building upon this idea, we extend structures from manifolds to structures on sheaves in the following way. For $\mathcal{F}, \mathcal{M} \in \mathrm{Shv}_{\infty}$, define

$$
\mathcal{F}(\mathcal{M}):=\operatorname{Shv}_{\infty}(\mathcal{M}, \mathcal{F}) \in \infty-\mathrm{Gpd} .
$$

When $M$ is a manifold, we recover the usual definition of $\mathcal{F}(M)$. If $\mathcal{F}(X) \in n$-Gpd for all $X$, then $\mathcal{F}(\mathscr{M}) \in n$-Gpd as well.

Example 2.9 (Equivariant forms). There is a natural isomorphism $\Omega_{G}(M) \cong \Omega\left(\mathcal{E}_{\nabla} G \times{ }_{G} M\right)$ $[\mathrm{FH}]$, which we now explain. The cochain complex $\left(\Omega_{G}^{*}(M), d_{G}\right)$ of equivariant differential forms is defined using the Weil model as the basic subcomplex

$$
\Omega_{G}(M):=\left(S \mathfrak{g}^{*} \otimes \Lambda \mathfrak{g}^{*} \otimes \Omega(M)\right)_{\text {bas }}, \quad\left|S^{1} \mathfrak{g}^{*}\right|=2, \quad\left|\Lambda^{1} \mathfrak{g}^{*}\right|=1,
$$


and for compact $G$ there is a natural de Rham isomorphism

$$
H^{n}\left(\Omega_{G}^{*}(M), d_{G}\right) \stackrel{\cong}{\longrightarrow} H_{G}^{n}(M ; \mathbb{R}):=H^{n}\left(E G \times_{G} M ; \mathbb{R}\right) .
$$

If $(P, \Theta) \in \operatorname{Bun}_{G, \nabla}(X)$ with curvature $\Omega$, the Weil homomorphism induces a natural map

$$
\begin{aligned}
& \Theta^{*}: \Omega_{G}^{n}(P)=\left(S \mathfrak{g}^{*} \otimes \Lambda \mathfrak{g}^{*} \otimes \Omega(P)\right)_{\mathrm{bas}}^{n} \longrightarrow \Omega^{n}(P)_{\mathrm{bas}} \cong \Omega^{n}(X) \\
& \alpha \otimes \beta \otimes \gamma \longmapsto \alpha(\Omega) \wedge \beta(\Theta) \wedge \gamma .
\end{aligned}
$$

By the Yoneda Lemma, a differential form $\omega \in \Omega^{n}(X)$ is equivalent to a map $X \stackrel{\omega}{\rightarrow} \Omega^{n}$. For $M \in G$-Man, it is therefore natural to define

$$
\Omega^{n}\left(\mathcal{E}_{\nabla} G \times_{G} M\right):=\operatorname{Shv}_{\infty}\left(\mathcal{E}_{\nabla} G \times_{G} M, \Omega^{n}\right) \in \operatorname{Set} \subset \infty-G p d .
$$

Unpackaging this, a form $\omega \in \Omega^{n}\left(\mathcal{E}_{\nabla} G \times_{G} M\right)$ assigns to every $X \stackrel{(P, \Theta, f)}{\longrightarrow} \mathcal{E}_{\nabla} G \times{ }_{G} M$ a differential form $\omega(P, \Theta, f) \in \Omega^{n}(X)$; since $\Omega^{n}(X)$ has no non-trivial morphisms, a map of principal $G$-bundles $P^{\prime} \stackrel{\varphi}{\rightarrow} P$ gives an equality of differential forms

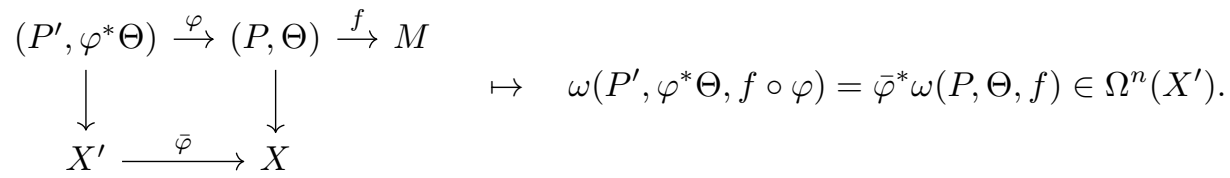

In $[\mathrm{FH}]$, Freed-Hopkins show the natural map induced by the Weil homomorphism gives an isomorphism, or bijection of sets,

$$
\Omega_{G}^{n}(M) \stackrel{\cong}{\rightarrow} \Omega^{n}\left(\mathcal{E}_{\nabla} G \times_{G} M\right) .
$$

Explicitly, a form $\omega \in \Omega_{G}^{n}(M)$ evaluates on $(P, \Theta, f) \in\left(\mathcal{E}_{\nabla} G \times_{G} M\right)(X)$ as the composition

$$
\Omega_{G}^{n}(M) \stackrel{f^{*}}{\longrightarrow} \Omega_{G}^{n}(P) \stackrel{\Theta^{*}}{\longrightarrow} \Omega^{n}(X),
$$

with $\omega(P, \Theta, f):=\Theta^{*} f^{*} \omega$. The familiar equivariant differential $d_{G}$ is induced by the universal map between sheaves $\Omega^{n} \stackrel{d}{\rightarrow} \Omega^{n+1}$. See [GS] or [Red1, Appendix A] for further details of equivariant de Rham theory.

Remark 2.13. One can equivalently use the Cartan model $\left(S \mathfrak{g}^{*} \otimes \Omega(M)\right)^{G}$ for $\Omega_{G}(M)$, since Mathai-Quillen give a natural isomorphism of cochain complexes (not merely a quasiisomorphism) between the Cartan and Weil models [MQ, Kal].

For $M \in G$-Man, let $G$-Bun $K, \nabla(M)$ denote the groupoid of $G$-equivariant principal $K$ bundles on $M$ with $G$-invariant connection; morphisms are $G$-equivariant bundle isomorphisms that preserve the connections. For brevity, we refer to $(Q, \Theta) \in G$-Bun $K, \nabla(M)$ as a $G$-equivariant $K$-connection on $M$. Equivariant connections always exist when $G$ is compact, meaning the forgetful map $G$-Bun $\operatorname{Bu}_{K}(M) \rightarrow G$-Bun $K(M)$ is surjective on objects.

Suppose that $P \in G$-Man has a free $G$-action, so that we can regard $P \in \operatorname{Bun}_{G}(X)$. Any $Q \in G$-Bun $K(P)$ naturally descends to a bundle $Q / G \in \operatorname{Bun}_{K}(X)$. However, a $K$-connection $\Theta_{Q}$ on $Q$ will descend to $Q / G$ if and only if it is $G$-basic. If $\left(P, \Theta_{P}\right) \in \operatorname{Bun}_{G, \nabla}(X)$, then there is a canonical way to modify the connection on $Q$ by $\Theta_{P}$, producing a $G$-basic connection

$$
\left(1-\iota_{\Theta_{P}}\right) \Theta_{Q} \in \Omega^{1}(Q, \mathfrak{k})_{G \text {-bas }} \cong \Omega^{1}(Q / G ; \mathfrak{k}),
$$

as explained in [Red1, Section 4.2] and [Red2, Section 3]. This connection form on $Q / G$ equals the image of $\Theta_{G} \in \Omega_{G}^{1}(Q ; \mathfrak{k})$, the $G$-equivariant extension of $\Theta_{Q}$, under the Weil 
homomorphism (2.10). Reusing the same symbol, for any $\left(P, \Theta_{P}\right) \in \operatorname{Bun}_{G, \nabla}(X)$ we denote the natural descent functor by

$$
G-\operatorname{Bun}_{K, \nabla}(P) \stackrel{\Theta_{P}^{*}}{\longrightarrow} \operatorname{Bun}_{K, \nabla}(X) .
$$

Example 2.15 (Equivariant connections). The construction (2.14) naturally induces a functor $G$-Bun $K, \nabla(M) \rightarrow \operatorname{Shv}_{\infty}\left(\mathcal{E}_{\nabla} G \times_{G} M, \mathcal{B}_{\nabla} K\right)$, as explained in both [Red1, Section 4.2] and [Fre]. For $\left(Q, \Theta_{Q}\right) \in G$-Bun $K, \nabla(M)$, define $\left(\mathcal{E}_{\nabla} G \times_{G} M\right)(X) \rightarrow\left(\mathcal{B}_{\nabla} K\right)(X)$ for every test manifold $X$ by

$$
\begin{array}{ll}
\left(P, \Theta_{P}\right) \stackrel{f}{\longrightarrow} M \\
\quad \downarrow \\
\quad X
\end{array} \quad \Theta_{P}^{*}\left(f^{*}\left(Q, \Theta_{Q}\right)\right) \in \operatorname{Bun}_{K, \nabla}(X),
$$

using the composition

$$
G-\operatorname{Bun}_{K, \nabla}(M) \stackrel{f^{*}}{\longrightarrow} G-\operatorname{Bun}_{K, \nabla}(P) \stackrel{\Theta_{P}^{*}}{\longrightarrow} \operatorname{Bun}_{K, \nabla}(X) .
$$

This construction establishes a functor between groupoids

$$
G-\operatorname{Bun}_{K, \nabla}(M) \stackrel{\cong}{\rightleftarrows} \operatorname{Bun}_{K, \nabla}\left(\mathcal{E}_{\nabla} G \times_{G} M\right):=\operatorname{Shv}_{\infty}\left(\mathcal{E}_{\nabla} G \times_{G} M, \mathcal{B}_{\nabla} K\right),
$$

which was proven to be an equivalence in [Red2] through the construction of an explicit inverse functor. Furthermore, consider the map $\mathcal{E}_{\nabla} G \times_{G} M \rightarrow \mathcal{B}_{\nabla} K$ induced by a specific $(Q, \Theta) \in G$-Bun $K, \nabla(M)$. As shown in [Red1, Theorem 5.3] and [Fre, Theorem 8], the induced map on differential forms

$$
\left(S^{n} \mathfrak{k}^{*}\right)^{K} \cong \Omega^{2 n}\left(\mathcal{B}_{\nabla} K\right) \longrightarrow \Omega^{2 n}\left(\mathcal{E}_{\nabla} G \times_{G} M\right) \cong \Omega_{G}^{2 n}(M)
$$

recovers the familiar equivariant Chern-Weil homomorphism of Berline-Vergne [BV].

Example 2.18 (Gerbes). When working with gerbes, we always consider gerbes with band $S^{1}$. Let $\operatorname{Grb}_{\nabla}(X) \in 2$-Gpd be the 2-groupoid of bundle gerbes with connection on $X$, as defined in [Wal1] and described in Section 3. As explained in [NS, Section 4.1], bundle gerbes satisfy the descent condition (2.2), and we denote the corresponding 2-stack by $\mathcal{B}_{\nabla}^{2} S^{1} \in \operatorname{Shv}_{\infty}$, where

$$
\left(\mathcal{B}_{\nabla}^{2} S^{1}\right)(X):=\operatorname{Grb}_{\nabla}(X) \in 2-\mathrm{Gpd} \subset \infty \text {-Gpd. }
$$

When $M \in G$-Man, the discussion of equivariant connections in Example 2.15 suggests that a "correct" definition of $G$-equivariant gerbe connections on $M$ is given by $\operatorname{Grb}_{\nabla}\left(\mathcal{E}_{\nabla} G \times_{M} G\right):=$ $\operatorname{Shv}_{\infty}\left(\mathcal{E}_{\nabla} G \times_{G} M, \mathcal{B}_{\nabla}^{2} S^{1}\right)$. This claim is what we ultimately prove in Theorems 4.15 and 4.16 .

2.3. Cohomology and differential cohomology. Using sheaves of $\infty$-groupoids on manifolds allows us to easily work with cohomology and differential cohomology. In doing so, we often use the Dold-Kan equivalence between non-negatively graded chain complexes of abelian groups and simplicial abelian groups

$$
\Gamma: \mathrm{Ch}_{\geq 0} \stackrel{\simeq}{\longrightarrow} \mathrm{Ab}^{\Delta^{\mathrm{op}}} .
$$

Let $A$ be a sheaf of abelian Lie groups, viewed as a Set-valued sheaf; i.e. $A(X) \in \mathrm{Ab} \subset$ Set $\subset \infty$-Gpd for every $X \in$ Man. By placing $A$ in degree $n$ of a chain complex, using the Dold-Kan functor and sheafifying, one gets an object

$$
\mathbb{L}(\Gamma(A[-n]))=\mathbb{L}(\Gamma(\cdots \rightarrow 0 \rightarrow A \rightarrow 0 \rightarrow \cdots \rightarrow 0)) \in \operatorname{Shv}_{\infty} .
$$


For $\mathcal{M} \in \operatorname{Shv}_{\infty}$, define the sheaf cohomology with coefficients in $A$ by

$$
H_{\text {Shv }}^{n}(\mathcal{M} ; A):=\pi_{0} \operatorname{Shv}_{\infty}(\mathcal{M}, \mathbb{L}(\Gamma(A[-n]))) \in \mathrm{Ab} .
$$

If $M \in$ Man, then $H_{\text {Shv }}^{*}(M ; A)$ recovers the traditional sheaf cohomology groups [Bro, Lur].

When $A=A^{\delta}$ is a discrete group, then $\mathcal{K}(A, n):=\mathbb{L}(\Gamma(A[-n]))$ is a Shv $v_{\infty}$ analogue of an Eilenberg-MacLane space. Since $\mathcal{K}(A, n)$ is homotopy-invariant, the arguments and techniques from $[\mathrm{BNV}]$ imply natural isomorphisms

$$
\begin{aligned}
H_{\mathrm{Shv}}^{n}\left(\mathcal{E}_{\nabla} G \times{ }_{G} M ; A\right) & \cong H_{\mathrm{Shv}}^{n}\left(\mathcal{E} G \times{ }_{G} M ; A\right) \\
& \cong \pi_{0} \operatorname{Top}\left(E G \times_{G} M, K(A, n)\right) \cong H_{G}^{n}(M ; A),
\end{aligned}
$$

as proven in [Red1, Proposition 6.7].

The differential cohomology groups, also known as Cheeger-Simons differential characters [CS] or Deligne cohomology [Del, Bry1], can be defined in a similar way [BNV, FSS, HQ]. Following the notation from $[\operatorname{Red} 1]$, let $\widehat{\mathcal{K}}(\mathbb{Z}, n) \in \mathrm{Shv}_{\infty}$ be a homotopy pullback in the diagram

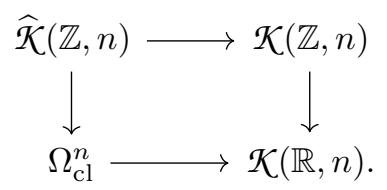

For $M \in G$-Man we define the differential equivariant cohomology groups as

$$
\widehat{H}_{G}^{n}(M):=\widehat{H}^{n}\left(\mathcal{E}_{\nabla} G \times_{G} M\right):=\pi_{0} \operatorname{Shv}_{\infty}\left(\mathcal{E}_{\nabla} G \times_{G} M, \widehat{\mathcal{K}}(\mathbb{Z}, n)\right) .
$$

Just as with traditional differential cohomology, the groups $\widehat{H}_{G}^{*}(M)$ sit in the following three short exact sequences [Red1].

$$
\begin{aligned}
0 \longrightarrow H_{G}^{n-1}(M ; \mathbb{R} / \mathbb{Z}) \longrightarrow \widehat{H}_{G}^{n}(M) \longrightarrow \Omega_{G}^{n}(M)_{\mathbb{Z}} \longrightarrow 0 \\
0 \longrightarrow \frac{\Omega_{G}^{n-1}(M)}{\Omega_{G}^{n-1}(M)_{\mathbb{Z}}} \longrightarrow \widehat{H}_{G}^{n}(M) \longrightarrow H_{G}^{n}(M ; \mathbb{Z}) \longrightarrow 0 \\
0 \longrightarrow \frac{H_{G}^{n-1}(M ; \mathbb{R})}{H_{G}^{n-1}(M ; \mathbb{R})_{\mathbb{Z}}} \longrightarrow \widehat{H}_{G}^{n}(M) \longrightarrow A_{G}^{n}(M) \longrightarrow 0
\end{aligned}
$$

Here $A^{n}(M)$ denotes the pullback (of sets) in the diagram

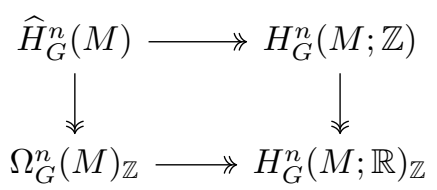

in which all maps are surjective. The subscript $\mathbb{Z}$ denotes the closed forms with integer periods and their image in de Rham cohomology. The groups $\widehat{H}_{G}^{n}(M)$ were also defined, independently and using different techniques, in $[\mathrm{KT}]$.

The equivalence $\mathcal{B}_{\nabla}^{2} S^{1} \simeq \mathbb{L}\left(\Gamma\left(S^{1} \stackrel{d}{\rightarrow} \Omega^{1} \stackrel{d}{\rightarrow} \Omega^{2}\right)\right) \simeq \widehat{\mathcal{K}}(\mathbb{Z}, 3)$, which is sometimes used as the definition of $\mathcal{B}_{\nabla}^{2} S^{1}$, immediately implies

$$
\pi_{0} \operatorname{Shv}_{\infty}\left(\mathcal{E}_{\nabla} G \times_{G} M, \mathcal{B}_{\nabla}^{2} S^{1}\right) \cong \widehat{H}_{G}^{3}(M) .
$$

This gives further evidence that $\operatorname{Grb}_{\nabla}\left(\mathcal{E}_{\nabla} G \times_{G} M\right)$ is a desirable model for equivariant gerbe connections. 


\section{The 2-Groupoid of EQuivariant Bundle Gerbe CONNECTIONS}

For a $G$-manifold $M \in G$-Man, we consider a geometrically defined 2-category $G$-Grb $\operatorname{Gr}_{\nabla}(M)$ of $G$-equivariant bundle gerbe connections, which is an abbreviated way of saying " $G$ equivariant bundle gerbes on $M$ with $G$-equivariant connection." Essentially, this is obtained from Waldorf's 2-groupoid $\operatorname{Grb}_{\nabla}(M)$ of bundle gerbes with connection (and invertible 1morphisms) [Wal1] and appending the adjective " $G$-equivariant" to everything; $G$-Grb $\operatorname{Gr}_{\nabla}(M)$ reduces to $\operatorname{Grb}_{\nabla}(M)$ when $G$ is the trivial group. The objects in $G$ - $\operatorname{Grb}_{\nabla}(M)$ were originally defined by Meinrenken [Mei] and further developed by Stiénon [Sti] and Tu-Xu [TX]. The key point is that the curving 2-form is an equivariant 2-form in $\Omega_{G}^{2}(Y)$, as opposed to other possible definitions [GSW1, Gom2]. We define the groupoid of morphisms in $G$-Grb $\operatorname{Gr}_{\nabla}(M)$ by adapting Waldorf's structures [Wal1] to the equivariant setting.

For $M \in G$-Man, define a $G$-cover to be a $G$-equivariant surjective submersion $Y \stackrel{\pi}{\rightarrow} M$; the fiber products $Y^{[p]}$ and projections $\pi_{i_{1} \cdots i_{k}}$ are naturally $G$-equivariant. Let $\delta$ denote the simplicial differential on $\Omega^{k}\left(Y^{[\bullet]}\right)$, which is given by the alternating sum of pullbacks. When $G$ is compact, the natural sequences

$$
\begin{gathered}
0 \rightarrow \Omega^{k}(M) \stackrel{\pi^{*}}{\rightarrow} \Omega^{k}(Y) \stackrel{\delta}{\rightarrow} \Omega^{k}\left(Y^{[2]}\right) \stackrel{\delta}{\rightarrow} \Omega^{k}\left(Y^{[3]}\right) \stackrel{\delta}{\rightarrow} \cdots \\
0 \rightarrow \Omega^{k}(M)^{G} \stackrel{\pi^{*}}{\rightarrow} \Omega^{k}(Y)^{G} \stackrel{\delta}{\rightarrow} \Omega^{k}\left(Y^{[2]}\right)^{G} \stackrel{\delta}{\rightarrow} \Omega^{k}\left(Y^{[3]}\right)^{G} \stackrel{\delta}{\rightarrow} \cdots \\
0 \rightarrow \Omega_{G}^{k}(M) \stackrel{\pi^{*}}{\rightarrow} \Omega_{G}^{k}(Y) \stackrel{\delta}{\rightarrow} \Omega_{G}^{k}\left(Y^{[2]}\right) \stackrel{\delta}{\rightarrow} \Omega_{G}^{k}\left(Y^{[3]}\right) \stackrel{\delta}{\rightarrow} \cdots
\end{gathered}
$$

are exact, as shown in [Sti, Lemma 3.3].

We also use $\delta$ to denote the analogous construction for $S^{1}$-bundles. In particular, for $L \in G-\operatorname{Bun}_{S^{1}}(Y)$,

and for $L \in G$-Bun $S^{1}\left(Y^{[2]}\right)$, then

$$
\delta L=\pi_{2}^{*} L \otimes \pi_{1}^{*} L^{-1} \in G-\operatorname{Bun}_{S^{1}}\left(Y^{[2]}\right),
$$

$$
\delta L=\pi_{23}^{*} L \otimes \pi_{13}^{*} L^{-1} \otimes \pi_{12}^{*} L \in G-\operatorname{Bun}_{S^{1}}\left(Y^{[3]}\right) .
$$

The construction $\delta$ also extends to $G$-equivariant principal $S^{1}$-bundles with $G$-invariant connection on $Y^{[k]}$, which we denote by $G$-Bun $S^{1}, \nabla\left(Y^{[k]}\right)$. We denote the trivial bundle (with trivial connection) by 1 , as it is naturally a unit under the tensor product.

Remark 3.4. Our notation implicitly uses the natural equivalence between principal $S^{1}$ bundles with connection and Hermitian line bundles with connection. For example, the product of two principal $S^{1}$-bundles is actually defined by the associated bundle construction, though we denote it by the tensor product. While our notation is technically imprecise, it is easier to read and should cause no confusion.

We now write the full definition of $G-\operatorname{Grb}_{\nabla}(M)$ for completeness, but almost all of Waldorf's arguments immediately generalize. This is because $G$-equivariant submersions and $G$-equivariant bundles pull back along $G$-equivariant maps, and the tensor product of two $G$-equivariant line bundles is again a $G$-equivariant line bundle. We will mainly reference Waldorf's proofs and only provide further details when some care is required.

\subsection{The definition.}

Definition 3.5 (Objects, c.f. [Wal1, Mei, Sti]). An object $\widehat{\mathcal{L}}=(Y, L, \nabla, B, \mu) \in G$-Grb $\nabla(M)$ consists of:

- A $G$-cover $Y \stackrel{\pi}{\rightarrow} M$,

- $(L, \nabla) \in G-\operatorname{Bun}_{S^{1}, \nabla}\left(Y^{[2]}\right)$, 
- $B \in \Omega_{G}^{2}(Y)$ satisfying $\operatorname{curv}_{G}(\nabla)=\pi_{2}^{*} B-\pi_{1}^{*} B \in \Omega_{G}^{2}\left(Y^{[2]}\right)$,

- and an isomorphism $\mu: \pi_{12}^{*}(L, \nabla) \otimes \pi_{23}^{*}(L, \nabla) \rightarrow \pi_{13}^{*}(L, \nabla)$ in $G$-Bun ${ }_{S^{1}, \nabla}\left(Y^{[3]}\right)$, called the bundle gerbe multiplication, that is associative in that the following diagram in $G$-Bun $S^{1}, \nabla\left(Y^{[4]}\right)$ commutes.

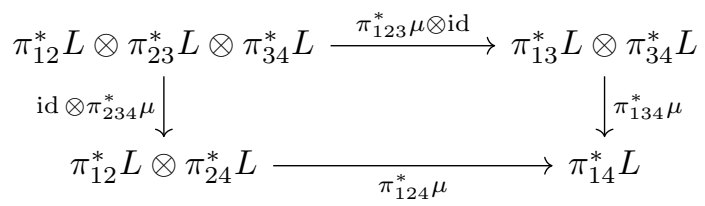

Remark 3.6. Readers feeling overwhelmed by the abundance of pullbacks can suppress them by writing $\pi_{i j}^{*}(L, \nabla)$ as $\left(L_{i j}, \nabla^{i j}\right)$. The key properties are then written $\operatorname{as} \operatorname{curv}_{G}\left(\nabla^{i j}\right)=$ $B_{j}-B_{i}, \mu: L_{i j} \otimes L_{j k} \rightarrow L_{i k}$, and $\mu\left(\mu\left(L_{i j} \otimes L_{j k}\right) \otimes L_{k l}\right)=\mu\left(L_{i j} \otimes \mu\left(L_{j k} \otimes L_{k l}\right)\right)$.

Remark 3.7. The isomorphism $\mu$ is equivalent to $\mu: \delta(L, \nabla) \rightarrow 1$ satisfying $\delta \mu \cong$ id under the natural isomorphism $\delta(\delta(L, \nabla)) \cong 1$.

Definition 3.8 (1-morphisms, c.f. [Wal1]). For $\widehat{\mathcal{L}}_{i}=\left(Y_{i}, L_{i}, \nabla_{i}, B_{i}, \mu_{i}\right) \in G$-Grb $\nabla(M)$, an isomorphism $\widehat{\mathcal{L}_{1}} \stackrel{\widehat{\mathcal{K}}}{\longrightarrow} \widehat{\mathcal{L}}_{2}$, denoted $\widehat{\mathcal{K}}=\left(Z, K, \nabla_{K}, \alpha\right) \in G$-Grb ${ }_{\nabla}\left(\widehat{\mathcal{L}}_{1}, \widehat{\mathcal{L}}_{2}\right)$, consists of:

- a $G$-cover $\zeta: Z \rightarrow Y_{1} \times_{M} Y_{2}$,

- $\left(K, \nabla_{K}\right) \in G-\operatorname{Bun}_{S^{1}, \nabla}(Z)$ such that $\operatorname{curv}_{G}\left(\nabla_{K}\right)=\zeta^{*}\left(B_{2}-B_{1}\right) \in \Omega_{G}^{2}(Z)$,

- and an isomorphism $\alpha:\left(L_{1}, \nabla_{1}\right) \otimes \zeta_{2}^{*}\left(K, \nabla_{K}\right) \rightarrow \zeta_{1}^{*}\left(K, \nabla_{K}\right) \otimes\left(L_{2}, \nabla_{2}\right)$ in $G$-Bun $S_{S^{1}, \nabla}\left(Z \times_{M}\right.$ $Z)$ that is compatible with $\mu_{1}$ and $\mu_{2}$, in that the following diagram in $G$-Bun ${ }_{S^{1}, \nabla}\left(Z \times{ }_{M}\right.$ $Z \times{ }_{M} Z$ ) commutes.

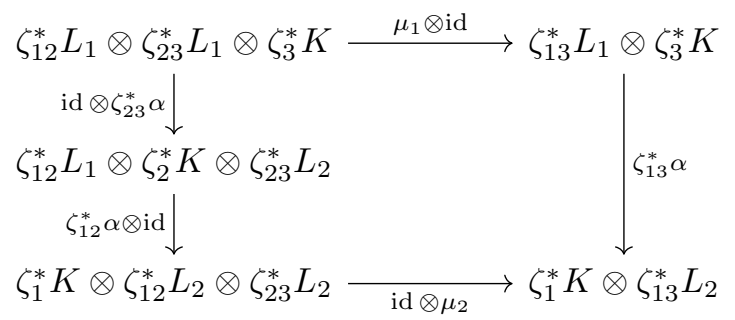

Remark 3.9. Throughout the paper, we avoid writing pullbacks when they are obvious so as not to further complicate notation. For example, in the above definition we use $L_{i} \in$ $G$-Bun $S_{S^{1}, \nabla}\left(Z \times_{M} Z\right)$ to denote the bundle obtained by pulling back $L_{i} \in G$-Bun ${ }_{S^{1}, \nabla}\left(Y_{i}^{[2]}\right)$ along the obvious map $Z \times{ }_{M} Z \rightarrow Y_{i}^{[2]}$.

Remark 3.10. The 1-morphisms in [Wal1] are more general than those in Definition 3.8. We restrict restrict ourselves to Waldorf's invertible morphisms, which are given by vector bundles of rank one. An analog of Waldorf's "more morphisms" category can be obtained by letting $(K, \nabla) \in G-\operatorname{Bun}_{U(n), \nabla}(M)$, but we do not consider them in this paper.

Definition 3.11 (2-morphisms, c.f. [Wal1]). For $\widehat{\mathcal{K}}_{1}, \widehat{\mathcal{K}}_{2} \in G$-Grb $\nabla\left(\widehat{\mathcal{L}}_{1}, \widehat{\mathcal{L}}_{2}\right)$, a 2-morphism $\widehat{\mathcal{J}}: \widehat{\mathcal{K}}_{1} \Rightarrow \widehat{\mathcal{K}}_{2}$ in $G-\operatorname{Grb}_{\nabla}(M)$, which can also be viewed as a 1-morphism in the groupoid $G-\operatorname{Grb}_{\nabla}\left(\widehat{\mathcal{L}}_{1}, \widehat{\mathcal{L}}_{2}\right)$, is denoted $\widehat{\mathcal{J}}=(W, \beta)$ and is an equivalence class of the following:

- a $G$-equivariant surjective submersion $\omega: W \rightarrow Z_{1} \times{ }_{\left(Y_{1} \times{ }_{M} Y_{2}\right)} Z_{2}$, 
- and an isomorphism $\beta:\left(K_{1}, \nabla_{1}\right) \rightarrow\left(K_{2}, \nabla_{2}\right)$ in $G$-Bun $S_{S^{1}, \nabla}(W)$ compatible with $\alpha_{1}$ and $\alpha_{2}$, in the sense that the following diagram in $G$-Bun $S_{S^{1}, \nabla}\left(W \times_{M} W\right)$ commutes.

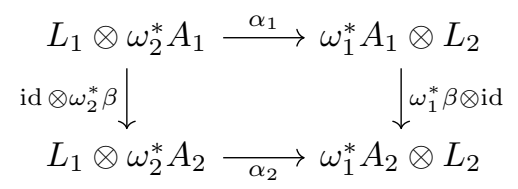

Two 2-morphisms $\widehat{\mathcal{J}}_{i}=\left(W_{i}, \beta_{i}\right): \widehat{\mathcal{K}} \Rightarrow \widehat{\mathcal{K}}^{\prime}$ are equivalent if there exists $G$-covers $\pi_{i}: X \rightarrow W_{i}$ for $i=1,2$ such that $\omega_{1} \circ \pi_{1}=\omega_{2} \circ \pi_{2}$ and $\pi_{1}^{*} \beta_{1}=\pi_{2}^{*} \beta_{2}$.

Morphisms can be easily composed using pullbacks, since the definitions of morphisms include the additional datum of a $G$-cover. These details are carefully discussed in [Wal1, Section 1], and they immediately generalize to the equivariant setting. Composition in the groupoid $G$-Grb $\operatorname{Gr}_{\nabla}\left(\widehat{\mathcal{L}}_{1}, \widehat{\mathcal{L}}_{2}\right)$, also referred to as vertical composition and denoted by $\bullet$, is defined by the following. If $\widehat{\mathcal{J}}: \widehat{\mathcal{K}}_{1} \Rightarrow \widehat{\mathcal{K}}_{2}$ and $\widehat{\mathcal{J}}^{\prime}: \widehat{\mathcal{K}}_{2} \Rightarrow \widehat{\mathcal{K}}_{3}$, then $\widehat{\mathcal{J}}^{\prime} \bullet \widehat{\mathcal{J}}: \widehat{\mathcal{K}}_{1} \Rightarrow \widehat{\mathcal{K}}_{3}$ is given by the $G$-cover $\widetilde{W}=W \times_{Z_{2}} W^{\prime} \rightarrow Z_{1} \times_{Y_{1} \times_{M} Y_{2}} Z_{3}$ and isomorphism $\beta^{\prime} \circ \beta: K_{1} \rightarrow$ $K_{3}$ in $G$-Bun $S_{S^{1}, \nabla}(\widetilde{W})$. The horizontal composition of 1 -morphisms in $G$-Grb $\operatorname{Gr}_{\nabla}(M)$ is defined in a similar way. For $\widehat{\mathcal{K}} \in G$-Grb $\nabla\left(\widehat{\mathcal{L}}_{1}, \widehat{\mathcal{L}}_{2}\right), \widehat{\mathcal{K}}^{\prime} \in G-G^{-} b_{\nabla}\left(\widehat{\mathcal{L}}_{2}, \widehat{\mathcal{L}}_{3}\right)$, the composition $\widehat{\mathcal{K}}^{\prime} \circ \widehat{\mathcal{K}}=(\widetilde{Z}, \widetilde{K}, \widetilde{\nabla}, \widetilde{\alpha}) \in G-G_{b}\left(\widehat{\mathcal{L}}_{1}, \widehat{\mathcal{L}}_{3}\right)$ is defined by $\widetilde{Z}:=Z \times_{Y_{2}} Z^{\prime} \rightarrow Y_{1} \times_{M} Y_{3}$, with $(\widetilde{K}, \widetilde{\nabla})=\left(K, \nabla_{K}\right) \otimes\left(K^{\prime}, \nabla_{K^{\prime}}\right)$ and with isomorphism $\widetilde{\alpha}=\left(\operatorname{id}_{\zeta_{1}^{*} K} \otimes \alpha^{\prime}\right) \circ\left(\alpha \otimes \operatorname{id}_{\zeta_{2}^{\prime *} K^{\prime}}\right)$ in $G$-Bun $S_{S^{1}, \nabla}\left(\widetilde{Z} \times_{M} \widetilde{Z}\right)$. Horizontal composition of 2-morphisms is defined as well, and we refer the interested reader to [Wal1, pp. 250-1] for full details.

By ignoring all the connections and differential forms, we may similarly define $G$ - $\operatorname{Grb}(M)$, the 2-groupoid of $G$-equivariant gerbes without connection. There is a natural forgetful functor

$$
\begin{aligned}
G-\operatorname{Grb}_{\nabla}(M) & \longrightarrow G-\operatorname{Grb}(M) \\
\widehat{\mathcal{L}}=(Y, L, \nabla, B, \mu) & \longmapsto \mathcal{L}=(Y, L, \mu),
\end{aligned}
$$

which we denote by eliminating the hat ${ }^{-}$symbol. By following the same arguments from [Wal1, Section 1], we conclude the following.

Theorem 3.12 (c.f. [Wal1]). If $M \in G$-Man, then $G-G_{r b}(M)$ and $G-G r b(M)$ are welldefined 2-groupoids.

3.2. Basic properties. It is evident that a $G$-equivariant map $M_{1} \stackrel{f}{\rightarrow} M_{2}$ induces functors

$$
\begin{gathered}
G-\operatorname{Grb}_{\nabla}\left(M_{2}\right) \stackrel{f^{*}}{\longrightarrow} G-\operatorname{Grb}_{\nabla}\left(M_{1}\right), \\
G-\operatorname{Grb}\left(M_{2}\right) \stackrel{f^{*}}{\longrightarrow} G-\operatorname{Grb}\left(M_{1}\right),
\end{gathered}
$$

given by pulling back all relevant $G$-equivariant structures along $G$-equivariant maps.

The exact sequence (3.2), which assumes $G$ is compact, implies that every $\mathcal{L} \in G$-Grb $(M)$ admits a $G$-equivariant connection [Sti, Propostion 3.2]. In other words, the forgetful functor $G-\operatorname{Grb}_{\nabla}(M) \rightarrow G$-Grb $(M)$ is surjective on objects. Furthermore, the exact sequence (3.3) implies that any $\widehat{\mathcal{L}} \in G-\operatorname{Grb}_{\nabla}(M)$ has a unique equivariant curvature $\operatorname{curv}_{G}(\widehat{\mathcal{L}}) \in \Omega_{G}^{3}(M)$ defined by the property

$$
\pi^{*} \operatorname{curv}_{G}(\widehat{\mathcal{L}})=d_{G} B \in \Omega_{G}^{3}(Y)
$$


and that $d_{G} \operatorname{curv}_{G}(\widehat{\mathcal{L}})=0 \in \Omega_{G}^{4}(M)$ [Sti, Lemma 3.3]. As one would expect, the equivariant curvature depends only on the isomorphism class of $\widehat{\mathcal{L}}$.

Proposition 3.15. If $\widehat{\mathcal{L}}_{1}, \widehat{\mathcal{L}}_{2} \in G-\mathrm{Grb}_{\nabla}(M)$ are isomorphic, then

$$
\operatorname{curv}_{G}\left(\widehat{\mathcal{L}}_{1}\right)=\operatorname{curv}_{G}\left(\widehat{\mathcal{L}}_{2}\right) \in \Omega_{G}^{3}(M) .
$$

Proof. Assume there is an isomorphism $\widehat{\mathcal{K}}: \widehat{\mathcal{L}}_{1} \rightarrow \widehat{\mathcal{L}}_{2}$, as described in Definition 3.8. The $G$ equivariant surjective submersions $Z \rightarrow Y_{i} \rightarrow M$ induce injective maps $\Omega_{G}(M) \rightarrow \Omega_{G}\left(Y_{i}\right) \rightarrow$ $\Omega_{G}(Z)$, which we suppress in the following calculation:

$$
\operatorname{curv}_{G}\left(\widehat{\mathcal{L}}_{2}\right)-\operatorname{curv}_{G}\left(\widehat{\mathcal{L}}_{1}\right)=d_{G}\left(B_{2}-B_{1}\right)=d_{G} \operatorname{curv}_{G}\left(\nabla^{K}\right)=0 \in \Omega_{G}^{3}(Z) .
$$

The first equality comes from the definition of equivariant gerbe curvature in (3.14). The second equality is in Definition 3.8 of 1-morphisms. The last equality follows because the equariant curvature of an equivariant $S^{1}$-bundle connection is equivariantly closed.

There is a canonical trivial gerbe $\mathcal{I} \in G$ - $\operatorname{Grb}(M)$ given by $Y=M, L=1$, and $\mu \cong$ id under the canonical identification $1 \otimes 1 \cong 1$. Using the same $G$-cover id: $M \rightarrow M$, any $B \in$ $\Omega_{G}^{2}(M)$ satisfies $\delta B=0=\operatorname{curv}_{G}(1)$, where $1 \in G$-Bun $S^{1}, \nabla(M)$ denotes the trivial bundle with trivial connection. Hence, any $B \in \Omega_{G}^{2}(M)$ defines $\widehat{\mathcal{I}}_{B}:=(M, 1, B$, id $) \in G$-Grb $\operatorname{Gr}_{\nabla}(M)$ with curvature $\operatorname{curv}_{G}\left(\widehat{\mathcal{I}}_{B}\right)=d_{G} B \in \Omega_{G}^{3}(M)$ and with underlying topological gerbe $\mathcal{I}$. The trivial gerbe with trivial connection is then denoted $\widehat{\mathcal{I}}_{0} \in G$-Grb $\nabla(M)$.

The natural Picard structure on the category of complex lines, given by the tensor product and the dual, naturally makes the category of $S^{1}$-bundles into a Picard groupoid and the category of gerbes into a Picard 2-groupoid. These structures naturally carry over to the equivariant setting, and the same proofs that Waldorf gives in [Wal1] show that $G-\mathrm{Grb}_{\nabla}(M)$ is a symmetric monoidal 2-category in which all 1- and 2-morphisms are invertible, and all objects are invertible under the tensor product (symmetric monoidal) structure. The curvature 3 -forms are additive with respect to tensor product,

$$
\operatorname{curv}_{G}\left(\widehat{\mathcal{L}}_{1} \otimes \widehat{\mathcal{L}}_{2}\right)=\operatorname{curv}_{G}\left(\widehat{\mathcal{L}}_{1}\right)+\operatorname{curv}_{G}\left(\widehat{\mathcal{L}}_{2}\right) .
$$

The tensor units are $\widehat{\mathcal{I}}_{0} \in G-\operatorname{Grb}_{\nabla}(M)$ and $\mathcal{I} \in G$-Grb $(M)$. In particular, there is a natural adjunction

$$
G-G r b_{\nabla}\left(\widehat{\mathcal{L}}_{1}, \widehat{\mathcal{L}}_{2}\right) \stackrel{\cong}{\rightrightarrows} G-\operatorname{Grb}_{\nabla}\left(\widehat{\mathcal{L}}_{0}, \widehat{\mathcal{L}}_{1}^{-1} \otimes \widehat{\mathcal{L}}_{2}\right)
$$

and a canonical trivialization $\widehat{\mathcal{I}}_{0} \stackrel{\cong}{\rightarrow} \widehat{\mathcal{L}}^{-1} \otimes \widehat{\mathcal{L}}$, which gives the natural equivalence of groupoids

$$
\operatorname{Aut}(\widehat{\mathcal{L}})=G-\operatorname{Grb}_{\nabla}(\widehat{\mathcal{L}}, \widehat{\mathcal{L}}) \cong G-\operatorname{Grb}_{\nabla}\left(\widehat{\mathcal{I}}_{0}, \widehat{\mathcal{L}}^{-1} \otimes \widehat{\mathcal{L}}\right) \cong G-\operatorname{Grb}_{\nabla}\left(\widehat{\mathcal{I}}_{0}, \widehat{\mathcal{I}}_{0}\right)=\operatorname{Aut}\left(\widehat{\mathcal{I}}_{0}\right) .
$$

3.3. Fiber product morphisms. For $\widehat{\mathcal{L}}_{1}, \widehat{\mathcal{L}}_{2} \in G-\operatorname{Grb}_{\nabla}(M)$, we consider the subgroupoid of fiber product morphisms $G$-Grb $\operatorname{Gr}_{\nabla}^{\mathrm{FP}}\left(\widehat{\mathcal{L}}_{1}, \widehat{\mathcal{L}}_{2}\right) \subset G-\mathrm{Grb}_{\nabla}\left(\widehat{\mathcal{L}}_{1}, \widehat{\mathcal{L}}_{2}\right)$. Objects are 1-morphisms $\widehat{\mathcal{K}}=\left(Z, K, \nabla_{K}, \alpha\right)$ whose equivariant surjective submersion $\zeta: Z \rightarrow Y_{1} \times_{M} Y_{2}$ is the identity; similarly, morphisms are $\widehat{\mathcal{J}}=(W, \beta)$ with $W \rightarrow Y_{1} \times_{M} Y_{2}$ the identity. Ignoring differential data, the same conditions also define $G$-Grb ${ }^{\mathrm{FP}}\left(\mathcal{L}_{1}, \mathcal{L}_{2}\right) \subset G$ - $\operatorname{Grb}\left(\mathcal{L}_{1}, \mathcal{L}_{2}\right)$. These fiber product morphisms, at least in the non-equivariant case, are often called stable isomorphisms [Ste].

In [Wal1, Theorem 2.1] Waldorf shows that the inclusion $\operatorname{Grb}_{\nabla}^{\mathrm{FP}}\left(\widehat{\mathcal{L}}_{1}, \widehat{\mathcal{L}}_{2}\right) \hookrightarrow \operatorname{Grb}_{\nabla}\left(\widehat{\mathcal{L}}_{1}, \widehat{\mathcal{L}}_{2}\right)$ is an equivalence of categories. The benefit is immediate: allowing arbitrary covers is a more natural definition of isomorphisms, but fiber product morphisms are easier to work with in practice. This equivalence of categories extends to the $G$-equivariant case, as stated 
in Proposition 3.22 below, and the proof is the same as Waldorf's argument in [Wal1]. We include some of these details, however, since the reader could potentially mistranslate one of Waldorf's arguments to the equivariant setting, as explained below.

Let $Z \stackrel{\zeta}{\rightarrow} M$ be a $G$-cover. Define the category $G$-Bun $\operatorname{Bu}_{S^{1}, \nabla}^{\text {desc }}(\zeta)$ as follows. Objects are triples $(K, \nabla, \mu)$, where $(K, \nabla) \in G$-Bun $S^{1}, \nabla(Z)$ and $\mu: \zeta_{1}^{*}(K, \nabla) \rightarrow \zeta_{2}^{*}(K, \nabla)$ is an isomorphism over $Z^{[2]}$ satisfying the cocycle identity $\zeta_{13}^{*} \mu=\zeta_{23}^{*} \mu \circ \zeta_{12}^{*} \mu$ on $Z^{[3]}$. Morphisms from $(K, \nabla, \mu)$ to $\left(K^{\prime}, \nabla^{\prime}, \mu^{\prime}\right)$ are isomorphisms $\alpha:(K, \nabla) \rightarrow\left(K^{\prime}, \nabla^{\prime}\right)$ such that the following diagram commutes.

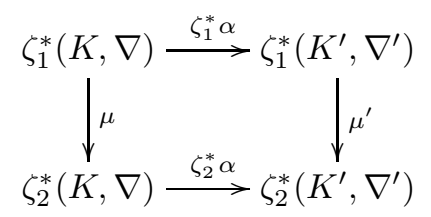

The following Lemma 3.19 is well-known in the non-equivariant case of principal bundles considered without connections; see Brylinski [Bry1, p.187]. The version in [Wal1] is not proven directly, but instead Waldorf utilizes the well-known fact that $S^{1}$-bundles with connection form a stack on the site of manifolds, something that $G$-Bun ${ }_{S^{1}, \nabla}(-)$ is not. The descent bundle, however, can be defined as a limit, and it will be $G$-equivariant if the original diagram was in $G$-Man. Since we could not find a reference for the equivariant version of descent, we include a full proof.

Lemma 3.19 (Descent for equivariant bundles). Let $Z \stackrel{\zeta}{\rightarrow} M$ be a $G$-cover. The functor

$$
\zeta^{*}: G-\operatorname{Bun}_{S^{1}, \nabla}(M) \rightarrow G-\operatorname{Bun}_{S^{1}, \nabla}^{\mathrm{desc}}(\zeta)
$$

is an equivalence of groupoids.

Proof. We shall prove that the functor $\zeta^{*}$ is essentially surjective and fully faithful. We verify essential surjectivity by taking an object in the descent groupoid and defining a $G$ equivariant $S^{1}$-bundle with connection over $M$ through a quotient construction using the descent isomorphism. For any $(K, \theta, \mu) \in G$-Bun $\operatorname{Sesc}_{S^{1}, \nabla}^{\text {desc }}(\zeta)$ we define a relation $\sim$ on $K$ as follows: For $k \in K_{z_{1}}$ and $k^{\prime} \in K_{z_{2}}, k \sim k^{\prime}$ if $\mu\left(\left(z_{1}, z_{2}\right), k\right)=\left(\left(z_{1}, z_{2}\right), k^{\prime}\right)$. Since $\mu$ is an isomorphism of $S^{1}$-bundles satisfying the cocycle identity, the relation $\sim$ is an equivalence relation. We construct a $G$-equivariant $S^{1}$-bundle $S$ over $M$ as follows. Define $S:=K / \sim$ and topologize it by the map $K \rightarrow K / \sim$. The projection map $\pi: S \rightarrow M$ is defined by $[k] \mapsto \zeta \circ \pi_{K}(k)$ for any $[k] \in S$, which is clearly well-defined. Here $\pi_{K}$ is the projection map $K \rightarrow Z$. For any $x \in M, S^{1}$ acts on $S_{x}:=\pi^{-1}(x)$ by $[k] \cdot r:=[k \cdot r]$ for all $[k] \in S_{x}$ and $r \in S^{1}$, and the action is free and transitive, since $S^{1}$ acts on $K$ as such. To show $S$ is locally trivial, we take any $x \in M$ and an open neighborhood $U$ of $x$ such that $\zeta$ splits locally by a smooth (non-equivariant) map $\sigma: U \rightarrow Z$ and the image of $\sigma$ is properly contained in an open neighborhood $V$ of $\sigma(x)$ over which $K$ is locally trivial by a local trivialization $\varphi:\left.K\right|_{V} \rightarrow V \times S^{1}$. We define a map $\left.S\right|_{U} \rightarrow U \times S^{1}$ by $[k] \mapsto\left(\zeta \circ \pi_{K}(k),\left.\varphi\right|_{V \cap \sigma(U)}(k)\right)$. This map is well-defined because, from the construction of $\sigma$, there is only one representative $k$ of $[k]$ that has $\pi_{K}(k) \in \sigma(U)$, and it is clearly $S^{1}$-equivariant. We now endow a $G$-action on $S$ by $g \cdot[k]:=[g \cdot k]$ making $S \in G$-Bun $S^{1}(M)$ since $K, \mu$ and $\zeta$ are $G$-equivariant, and the action commutes with the right $S^{1}$-action on $S$.

Now we define a $G$-invariant connection $\Theta$ on $S$. Let $v_{1} \in T_{k_{1}} K$ and $v_{2} \in T_{k_{2}} K$, where $\pi_{K}\left(k_{i}\right)=z_{i}$ for $i=1,2$. We define a relation $v_{1} \sim_{*} v_{2}$ if there is a path $(\alpha(t), \gamma(t))$ in $\zeta_{1}^{*} K$ and a path $(\beta(t), \delta(t))$ in $\zeta_{2}^{*} K$ satisfying $\alpha(0)=\left(z_{1}, z_{2}\right)=\beta(0), \gamma(0)=k_{1}, \gamma^{\prime}(0)=v_{1}$, 
$\delta(0)=k_{2}$ and $\delta^{\prime}(0)=v_{2}$ such that $\mu_{*}\left(\gamma^{\prime}(0)\right)=\delta^{\prime}(0)$. Here $\mu_{*}$ denotes the derivative of $\mu$. The relation $\sim_{*}$ is an equivalence relation since $\sim$ is an equivalence relation and the push-forward is a functor. We define $\Theta \in \Omega^{1}(S)$ by $\Theta_{[k]}([v]):=\theta_{k}(v)$. This is well-defined: For any $v_{1} \sim_{*} v_{2}$ and $k_{1} \sim k_{2}$, we see that

$$
\Theta_{\left[k_{2}\right]}\left(\left[v_{2}\right]\right)=\theta_{k_{2}}\left(v_{2}\right)=\mu^{*} \theta_{k_{1}}\left(v_{1}\right)=\theta_{k_{1}}\left(v_{1}\right)=\Theta_{\left[k_{1}\right]}\left(\left[v_{1}\right]\right) .
$$

The third equality follows from the fact that $\mu$ is a connection preserving isomorphism. It is easy to see that $R_{r}^{*} \Theta=\operatorname{Ad}_{r^{-1}} \Theta$ and $\Theta(\rho(r))=r$ for $r \in \mathbb{R}$. Here $\rho_{[k]}(r)=([k] \cdot \exp (t \cdot r))^{\prime}(0)$. We also note that $\Theta$ is $G$-invariant: For any $g \in G$ and $v \in T_{k} K$ such that $v=\gamma^{\prime}(0)$,

$$
\begin{aligned}
\left(L_{g}^{*} \Theta\right)_{[k]}([v]) & =\Theta_{[g \cdot k]}\left(\left.\frac{d}{d t}\right|_{t=0} g \cdot[\gamma(t)]\right)=\Theta_{[g \cdot k]}\left(\left[L_{g_{*}}(v)\right]\right) \\
& =\theta_{g \cdot k}\left(L_{g_{*}}(v)\right)=\left(g^{*} \theta\right)_{k}(v) \stackrel{*}{=} \theta_{k}(v)=\Theta_{[k]}([v]) .
\end{aligned}
$$

The equality $*$ follows from the $G$-invariance of the connection form $\theta$.

We verify that $\zeta^{*}(S, \Theta)$ is isomorphic to $(K, \theta)$. First we note that the map

$$
\begin{aligned}
K & \stackrel{\varphi}{\rightarrow} \zeta^{*} S \\
k & \mapsto\left(\pi_{K}(k),[k]\right)
\end{aligned}
$$

is an isomorphism of $G$-equivariant $S^{1}$-bundles. Now we see that $\varphi^{*} \widetilde{\zeta}^{*} \Theta=\theta$. Here $\widetilde{\zeta}$ is a $G$-equivariant $S^{1}$-bundle map $\zeta^{*} S \rightarrow S$ covering $\zeta$. For $k$ and $v$ as above,

$$
\left(\varphi^{*} \widetilde{\zeta}^{*} \Theta\right)_{k}(v)=\left(\widetilde{\zeta}^{*} \Theta\right)_{\left(\pi_{K}(k),[k]\right)}([v])=\Theta_{[k]}([v])=\theta_{k} v .
$$

It is readily seen that the diagram as in (3.18) is commutative. Therefore the functor $\zeta^{*}$ is essentially surjective.

The functor $\zeta^{*}$ is obviously faithful. We show that it is full. For any $\alpha:\left(\left(\zeta^{*} S_{1}, \widetilde{\zeta}_{S_{1}}^{*} \theta_{1}\right), 1\right) \rightarrow$ $\left(\left(\zeta^{*} S_{2}, \widetilde{\zeta}_{S_{2}}^{*} \theta_{2}\right), 1\right)$ where $\widetilde{\zeta}_{S_{i}}^{*}: \zeta^{*} S_{i} \rightarrow S_{i}$ is the map covering $\zeta$, there exists some $\beta \in$ $\operatorname{Bun}_{S^{1}}(M)\left(S_{1}, S_{2}\right)$ such that $\zeta^{*} \beta=\alpha$ because of the above-mentioned special case in [Bry1]. Since $\alpha$ is $G$-equivariant, it follows that $\beta \in G$-Bun $S^{1}(M)\left(S_{1}, S_{2}\right)$. From $\zeta$ being a surjective submersion, for any $v \in T_{s} S_{1}$ with $s \in S_{1}$, there exists $\widetilde{v} \in T_{(z, s)} \zeta^{*} S_{1}$ with $\zeta(z)=\pi_{S_{1}}(s)$ such that $\left(\widetilde{\zeta}_{S_{1}}\right)_{*} \widetilde{v}=v$. Hence we see that $\theta_{1 s}(v)=\left(\widetilde{\zeta}_{S_{1}}^{*} \theta_{1}\right)_{(z, s)}(\widetilde{v})=\left(\alpha^{*} \widetilde{\zeta}_{S_{2}}^{*} \theta_{2}\right)_{(z, s)}(\widetilde{v})=$ $\left(\widetilde{\zeta}_{S_{1}}^{*} \beta^{*} \theta_{2}\right)_{(z, s)}(\widetilde{v})=\left(\beta^{*} \theta_{2}\right)_{s}(v)$ and thus $\beta \in G$-Bun $S^{1}, \nabla(M)\left(\left(S_{1}, \theta_{1}\right),\left(S_{2}, \theta_{2}\right)\right)$.

We need two lemmas which are enhancements of [Wal1, Lemmas 1.3 and 1.5] in the $G$-equivariant case with connection. Their proofs follow line-by-line from Waldorf.

Lemma 3.20. Let $\widehat{\mathcal{L}}=(Y, L, \nabla, B, \mu) \in G-\operatorname{Grb}_{\nabla}(M)$. There is a canonical isomorphism $t_{\mu}$ : $\Delta^{*} L \rightarrow 1$ of $G$-equivariant $S^{1}$-bundles over $Y$ satisfying $\pi_{1}^{*} t_{\mu} \otimes \mathrm{id}=\Delta_{112}^{*} \mu$ and $\mathrm{id} \otimes \pi_{2}^{*} t_{\mu}=$ $\Delta_{122}^{*} \mu$. Here $\Delta: Y \rightarrow Y \times_{M} Y$ is the diagonal map, and $\Delta_{112}, \Delta_{122}: Y^{[2]} \rightarrow Y^{[3]}$ duplicate the first and the second factors, respectively.

Lemma 3.21. For any $\widehat{\mathcal{K}}=\left(Z \stackrel{\zeta}{\rightarrow} P:=Y_{1} \times_{M} Y_{2}, K, \nabla_{K}, \alpha\right) \in G-G_{b} \nabla_{\nabla}(M)\left(\widehat{\mathcal{L}}_{1}, \widehat{\mathcal{L}}_{2}\right)$, there exists a canonical isomorphism $d_{\widehat{\mathcal{K}}}: \zeta_{1}^{*}\left(K, \nabla_{K}\right) \rightarrow \zeta_{2}^{*}\left(K, \nabla_{K}\right)$ such that $\left(\left(K, \nabla_{K}\right), d_{\widehat{\mathcal{K}}}\right) \in$ $G$-Bun $\operatorname{Sin}^{1}, \nabla(\zeta)$ and the diagram

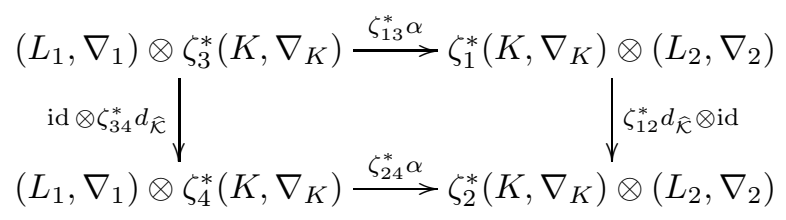


is commutative over $\left(Z \times_{P} Z\right) \times_{M}\left(Z \times_{P} Z\right)$.

Proposition 3.22. The natural inclusions

$$
\begin{aligned}
& G-\operatorname{Grb}^{\mathrm{FP}}\left(\mathcal{L}_{1}, \mathcal{L}_{2}\right) \stackrel{\simeq}{\longrightarrow} G-\operatorname{Grb}\left(\mathcal{L}_{1}, \mathcal{L}_{2}\right), \\
& G-\operatorname{Grb}_{\nabla}^{\mathrm{FP}}\left(\widehat{\mathcal{L}}_{1}, \widehat{\mathcal{L}}_{2}\right) \stackrel{\simeq}{\longrightarrow} G-\operatorname{Grb}_{\nabla}\left(\widehat{\mathcal{L}}_{1}, \widehat{\mathcal{L}}_{2}\right),
\end{aligned}
$$

are equivalences of categories.

Proof. We verify that the functor defined on $G-\operatorname{Grb}_{\nabla}^{\mathrm{FP}}\left(\widehat{\mathcal{L}}_{1}, \widehat{\mathcal{L}}_{2}\right)$ is essentially surjective. Given any $\widehat{\mathcal{K}}=\left(Z, K, \nabla_{K}, \alpha\right) \in G$-Grb $_{\nabla}(M)\left(\widehat{\mathcal{L}}_{1}, \widehat{\mathcal{L}}_{2}\right)$ with $G$-cover $Z \stackrel{\zeta}{\rightarrow} P:=Y_{1} \times_{M} Y_{2}$, by Lemma 3.21 , it follows that $\alpha$ is a morphism of the category $G$-Bun ${ }_{S^{1}, \nabla}^{\text {desc }}\left(\zeta^{2}\right)$ where $\zeta^{2}: Z \times{ }_{M} Z \rightarrow$ $P \times_{M} P$ is a $G$-cover. By Lemma 3.19 there exists $\left(S, \nabla_{S}\right) \in G$-Bun $S^{1}, \nabla(P)$ such that $\beta: \zeta^{*}\left(S, \nabla_{S}\right) \stackrel{\cong}{\rightarrow}\left(K, \nabla_{K}\right)$ and since $\alpha$ is a morphism of $G$-Bun $S_{S^{1}, \nabla}^{\text {desc }}\left(\zeta^{2}\right)$, by the same lemma there exists a morphism $\sigma$ in $G$-Bun $S^{1}, \nabla\left(P^{[2]}\right)$ that is pulled back to $\alpha$. Now we define a 1-morphism $\mathcal{S}_{\widehat{\mathcal{K}}} \in G$-Grb $\operatorname{Gr}_{\nabla}(M)\left(\widehat{\mathcal{L}}_{1}, \widehat{\mathcal{L}}_{2}\right)$ by $\left(P, S, \nabla_{S}, \sigma\right)$ with $G$-cover id ${ }_{P}$. Then there is a 2 morphism $\mathcal{S}_{\widehat{\mathcal{K}}} \Rightarrow \widehat{\mathcal{K}}$ defined by $(Z, \beta)$ with $G$-cover $\operatorname{id}_{Z}$ under the identification $Z \times_{P} P \cong Z$. The inclusion functor is clearly faithful, and we show that it is full. Let $\beta: \widehat{\mathcal{K}}_{1} \Rightarrow \widehat{\mathcal{K}}_{2}$ be a 2-morphism $\left(W, \beta_{W}\right)$ with $G$-cover $W \stackrel{\omega}{\rightarrow} P$. Since $Z \times_{P} Z \hookrightarrow Z \times_{M} Z$ is $G$-equivariant, the compatibility of $\omega$ with $\alpha_{i}$ of $\widehat{\mathcal{K}}_{i}$, over $Z \times_{M} Z$ implies $\omega_{1}^{*} \beta_{W}=\omega_{2}^{*} \beta_{W}$ over $Z \times_{P} Z$, and hence $\beta_{W}$ is a morphism of $G$-Bun $\operatorname{Susc}_{S^{1}, \nabla}^{\text {desc }}(\omega)$. By Lemma 3.19 there exists a morphism $\beta_{P}$ in $G$-Bun $S^{1}, \nabla(P)$ that is pulled back to $\beta_{W}$. Accordingly we have a 2 -morphism defined by the pair $\left(P, \beta_{P}\right)$ with $G$-cover $\operatorname{id}_{P}$ which is equivalent to the pair defining $\beta$.

3.4. Trivializations and isomorphism classes. Proposition 3.22 greatly simplifies the types of morphisms one must consider. First, the natural equivalence $G$-Grb $\left(\mathcal{L}_{1}, \mathcal{L}_{2}\right) \cong$ $G$-Grb $\left(\mathcal{I}, \mathcal{L}_{1}^{-1} \otimes \mathcal{L}_{2}\right)$, given by the Picard structure, tells us that to understand isomorphisms in $G$-Grb $(M)$ it suffices to understand isomorphisms $\mathcal{I} \rightarrow \mathcal{L}$, and thus it suffices to understand the fiber product trivializations $G$-Grb ${ }^{\mathrm{FP}}(\mathcal{I}, \mathcal{L})$.

Let us unpackage the data in an arbitrary $\mathcal{K}=(Z, K, \alpha) \in G$-Grb ${ }^{\mathrm{FP}}(\mathcal{I}, \mathcal{L})$. We may assume the $G$-cover $\zeta: Z \rightarrow M \times{ }_{M} Y \cong Y$ is the identity id: $Y \rightarrow Y$. Continuing, we have a bundle $K \in G$-Bun $S^{1}(Y)$ with isomorphism $\pi_{2}^{*} K \stackrel{\alpha}{\rightarrow} \pi_{1}^{*} K \otimes L$ in $G$-Bun $S_{S^{1}}\left(Y^{[2]}\right)$. Analogous to the description of $\mu$ in Remark 3.7, the isomorphism $\alpha$ is equivalent to an isomorphism $\alpha: \delta K \rightarrow L$. The condition that $\alpha$ is compatible with $\mu$ now means that

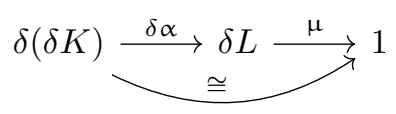

commutes; i.e. $\mu \circ(\delta \alpha)$ is the canonical isomorphism $\delta(\delta K) \cong 1$ in $G$-Bun $S_{S^{1}}\left(Y^{[3]}\right)$. A 2 -morphism between two such trivializations is an isomorphism $K \rightarrow K^{\prime}$ in $G$-Bun $S^{1}(Y)$ compatible with $\alpha, \alpha^{\prime}$.

Define $G$-Grbtriv $\operatorname{Gr}_{\nabla}(M)$ as the full subcategory of $G-\mathrm{Grb}_{\nabla}(M)$ consisting of objects $\widehat{\mathcal{L}}$ such that the underlying topological gerbe is isomorphic to the trivial gerbe $\mathcal{I} \cong \mathcal{L} \in G$-Grb $(M)$. This is the analog of a topologically trivializable $S^{1}$-bundle whose connection has non-trivial holonomy.

Previously, we discussed how any $B \in \Omega_{G}^{2}(M)$ determines $\widehat{\mathcal{I}}_{B} \in G$-Grbtriv $\nabla(M)$ given by the trivial gerbe but with curving 2-form $B$. We now show that this map is surjective on isomorphism classes of objects. Note that any $\left(K, \nabla_{K}\right) \in G$-Bun $S^{1}, \nabla(M)$ naturally determines a 1-morphism $\widehat{\mathcal{I}}_{B} \rightarrow \widehat{\mathcal{I}}_{B+\operatorname{curv}_{G}\left(\nabla_{K}\right)}$. In this way, the groupoid $G$-Bun $S^{1}, \nabla(M)$ 
acts on $\Omega_{G}^{2}(M)$, and we denote the induced action 2-groupoid as $\Omega_{G}^{2}(M) / / G$-Bun $S_{S^{1}, \nabla}(M)$. Explicitly, $\Omega_{G}^{2}(M)$ is the set of objects, and the groupoid of morphisms from $B_{1}$ to $B_{2}$ is the subgroupoid of $G$-Bun $S^{1}, \nabla(M)$ given by $(K, \nabla)$ with $\operatorname{curv}_{G}(\nabla)=B_{2}-B_{1}$.

We first point out a basic lemma whose proof is a simple homework problem.

Lemma 3.23. Suppose $G_{1} \rightarrow G_{2} \rightarrow G_{3}$ is an exact sequence of groups, and

$$
\begin{gathered}
A_{1} \longrightarrow f_{1} \\
\overbrace{\circlearrowleft} A_{2} \\
G_{1} \longrightarrow f_{2}
\end{gathered} A_{\circlearrowleft}^{A_{3}}
$$

are equivariant maps, where each $A_{i}$ is acted on freely and transitively by $G_{i}$. Then, the image $f_{2}\left(f_{1}\left(A_{1}\right)\right)$ is a single element $* \in A_{3}$; and, $a_{2} \in A_{2}$ is in the image of $f_{1}$ if and only if $f_{2}\left(a_{2}\right)=* \in A_{3}$.

Proposition 3.24. The natural functor

$$
\Omega_{G}^{2}(M) / / G-\operatorname{Bun}_{S^{1}, \nabla}(M) \stackrel{\widehat{\mathcal{I}}_{\bullet}}{\longrightarrow} G \text {-Grbtriv }_{\nabla}(M)
$$

is an equivalence of 2-groupoids and induces a natural isomorphism of abelian groups

$$
\frac{\Omega_{G}^{2}(M)}{\Omega_{G}^{2}(M)_{\mathbb{Z}}} \cong \pi_{0}\left(G-\operatorname{Grbtriv}_{\nabla}(M)\right) .
$$

Proof. We first we show that the functor is surjective on isomorphism classes of objects by proving that any $\widehat{\mathcal{L}}=\left(Y, L, \nabla^{L}, B, \mu\right) \in G-\operatorname{Grbtriv}_{\nabla}(M)$ is isomorphic to $\widehat{\mathcal{I}}_{\rho}$ for some $\rho \in \Omega_{G}^{2}(M)$. By definition, if $\widehat{\mathcal{L}} \in G$ - $\operatorname{Grbtriv}_{\nabla}(M)$ there exists a topological isomorphism $\mathcal{I} \stackrel{\mathcal{K}}{\rightarrow} \mathcal{L}$. As explained above, we use Proposition 3.22 to assume that $\mathcal{K}$ is given by a bundle $K \in G$-Bun $S^{1}, \nabla(Y)$ and an isomorphism

$$
\delta K \stackrel{\alpha}{\longrightarrow} L \quad \in G-\operatorname{Bun}_{S^{1}}\left(Y^{[2]}\right),
$$

where $\delta K$ denotes $\pi_{2}^{*} K \otimes\left(\pi_{1}^{*} K\right)^{-1}$. We now show the existence of a $G$-equivariant connection on $K$. Letting $\mathcal{A}(-)^{G}$ denote the affine space of $G$-invariant connections on an equivariant bundle, we have

$$
\begin{aligned}
\mathcal{A}(K)^{G} \stackrel{\delta}{\longrightarrow} \mathcal{A}(\delta K)^{G} \stackrel{\delta}{\longrightarrow} \mathcal{A}\left(\delta^{2} K\right)^{G} \cong \mathcal{A}(1)^{G} \\
\Omega^{1}(M)^{G} \longrightarrow \Omega^{1}(Y)^{G} \stackrel{\delta}{\longrightarrow} \Omega^{1}\left(Y^{[2]}\right)^{G} \stackrel{\delta}{\longrightarrow} \Omega^{1}\left(Y^{[3]}\right)^{G},
\end{aligned}
$$

where the top row is affine over the bottom row, and the bottom row is the exact sequence (3.3). By Lemma 3.23, an element $\nabla^{\delta K} \in \mathcal{A}(\delta K)^{G}$ is in the image of $\delta\left(\mathcal{A}(K)^{G}\right)$ if and only if it induces the trivial connection on $\delta(\delta K) \cong 1$, where the canonical isomorphism also equals $\mu \circ \delta \alpha$. Pulling back $\nabla^{L}$ via $\alpha$ gives $\left(\delta K, \alpha^{*} \nabla^{L}\right) \in G$-Bun $S^{1}, \nabla\left(Y^{[2]}\right)$. The natural isomorphism $\delta\left(L, \nabla^{L}\right) \stackrel{\mu}{\rightarrow} 1$ then implies implies that

$$
\delta\left(\alpha^{*} \nabla^{L}\right)=(\delta \alpha)^{*}\left(\delta \nabla^{L}\right)=(\delta \alpha)^{*}\left(\mu^{*} 1\right)=(\mu \circ \delta \alpha)^{*} 1 .
$$

Hence, there exists a (non-unique) connection $\nabla^{K}$ such that $\left(K, \nabla^{K}\right) \in G$-Bun $S_{S^{1}, \nabla}(Y)$ and $\delta\left(K, \nabla^{K}\right) \stackrel{\alpha}{\rightarrow}\left(L, \nabla^{L}\right) \in G$-Bun $S_{S^{1}, \nabla}\left(Y^{[2]}\right)$. Finally, consider $B-\operatorname{curv}_{G}\left(\nabla^{K}\right) \in \Omega_{G}^{2}(Y)$. Since $\delta\left(B-\operatorname{curv}_{G}\left(\nabla^{K}\right)\right)=\operatorname{curv}_{G}\left(\nabla^{L}\right)-\operatorname{curv}_{G}\left(\alpha^{*} \nabla^{L}\right)=0$, it follows that there exists a unique $\rho \in \Omega_{G}^{2}(M)$ such that $\pi^{*} \rho=B-\operatorname{curv}_{G}\left(\nabla^{K}\right)$. Therefore, there exists an isomorphism $\widehat{\mathcal{K}}: \widehat{\mathcal{I}}_{\rho} \rightarrow \widehat{\mathcal{L}}$ given by $\widehat{\mathcal{K}}=\left(Y, K, \nabla^{K}, \alpha\right)$.

We now consider the groupoid of morphisms. Any $(K, \nabla) \in G$-Bun $S_{S^{1}, \nabla}(M)$ that satisfies $\operatorname{curv}_{G}(\nabla)=B_{2}-B_{1} \in \Omega_{G}^{2}(M)$ clearly determines a 1-morphism, viewed as an object in the 
groupoid of morphisms $G-G_{r b}\left(\widehat{\mathcal{I}}_{B_{1}}, \widehat{\mathcal{I}}_{B_{2}}\right)$. To see that this is essentially surjective, we again will use Lemma 3.19. We may assume that an isomorphism $\widehat{\mathcal{I}}_{B_{1}} \rightarrow \widehat{\mathcal{I}}_{B_{2}}$ is isomorphic to one given by a $G$-equivariant $S^{1}$-connection $(K, \nabla) \in G$-Bun $S^{1}, \nabla(M)$ satisfying $\operatorname{curv}_{G}(\nabla)=$ $B_{2}-B_{1}$, together with an isomorphism $\alpha:(K, \nabla) \rightarrow(K, \nabla)$. The compatibility of $\alpha$ with $\mu_{1}=\mu_{2}=$ id implies that $\alpha \circ \alpha=\alpha$, and hence $\alpha=$ id (c.f. [Wal1, Section 3]). Therefore, any 1-morphism $\widehat{\mathcal{I}}_{B_{1}} \rightarrow \widehat{\mathcal{I}}_{B_{2}}$ is equivalent to some $(K, \nabla) \in G$-Bun $S_{S^{1}, \nabla}(M)_{B_{2}-B_{1}}$. Finally, for such 1-morphisms $\left(K_{i}, \nabla^{i}\right)$, the 2-morphisms $\left(K_{1}, \nabla^{1}\right) \Rightarrow\left(K_{2}, \nabla^{2}\right)$ in $G$-Grb $\operatorname{Fr}_{\nabla}^{\mathrm{FP}}\left(\widehat{\mathcal{I}}_{B_{1}}, \widehat{\mathcal{I}}_{B_{2}}\right)$ are precisely isomorphisms in $G$-Bun $S^{1}, \nabla(M)$.

Finally, the image of $\operatorname{curv}_{G}: G$-Bun $S^{1}, \nabla(M) \rightarrow \Omega_{G}^{2}(M)$ equals $\Omega_{G}^{2}(M)_{\mathbb{Z}}$. This fact, which we believe is fairly well-known, is implied by the isomorphism $\pi_{0}\left(G-\operatorname{Bun}_{S^{1}, \nabla}(M)\right) \cong \widehat{H}_{G}^{2}(M)$ [Red1, Proposition 5.10] and the fact that $\widehat{H}_{G}^{2}(M) \rightarrow \Omega_{G}^{2}(M)_{\mathbb{Z}}$ is surjective.

Corollary 3.25. The natural functors

$$
\begin{aligned}
& G-\operatorname{Bun}_{S^{1}, \nabla}(M)_{\text {flat }} \stackrel{\simeq}{\rightarrow} \operatorname{Aut}(\widehat{\mathcal{L}}), \\
& G-\operatorname{Bun}_{S^{1}}(M) \stackrel{\simeq}{\rightarrow} \operatorname{Aut}(\mathcal{L}),
\end{aligned}
$$

are equivalences of groupoids.

Proof. As noted in (3.17), the Picard structure gives a natural identification $\operatorname{Aut}(\widehat{\mathcal{L}}) \cong$ $\operatorname{Aut}\left(\widehat{\mathcal{I}}_{0}\right)$ for any $\widehat{\mathcal{L}} \in G$-Grb $\nabla(M)$. By Proposition 3.24 , Aut $\left(\widehat{\mathcal{I}}_{0}\right)$ is equivalent to the full subgroupoid of $G$-Bun $S_{S^{1}, \nabla}(M)$ consisting of objects $(K, \nabla)$ with equivariant curvature $\operatorname{curv}_{G}(\nabla)=0-0=0$, which is the groupoid $G-\operatorname{Bun}_{S^{1}, \nabla}(M)_{\text {flat }}$. Similarly, there is a natural identification $\operatorname{Aut}(\mathcal{L}) \cong \operatorname{Aut}(\mathcal{I})$ for any $\mathcal{L} \in G-\operatorname{Grb}(M)$. The same argument used in Proposition 3.24, but ignoring the differential forms and connection data, shows $\operatorname{Aut}(\mathcal{I})$ is equivalent to $G-\operatorname{Bun}_{S^{1}}(M)$.

Proposition 3.26. Let $\widehat{\mathcal{L}}_{1}, \widehat{\mathcal{L}}_{2} \in G-G b_{\nabla}(M)$. If there is an isomorphism of the underlying equivariant bundle gerbes without connection $\mathcal{L}_{1} \stackrel{\mathcal{K}}{\rightarrow} \mathcal{L}_{2} \in G-\operatorname{Grb}(M)$, then

$$
\left[\operatorname{curv}_{G}\left(\widehat{\mathcal{L}}_{1}\right)\right]=\left[\operatorname{curv}_{G}\left(\widehat{\mathcal{L}}_{2}\right)\right] \in H_{G}^{3}(M ; \mathbb{R}) .
$$

Proof. Let $\mathcal{K}: \mathcal{L}_{1} \rightarrow \mathcal{L}_{2}$ be an isomorphism, which is is equivalent to an isomorphism $\mathcal{I} \rightarrow$ $\mathcal{L}_{1}^{-1} \otimes \mathcal{L}_{2}$. By Proposition 3.24, there is an isomorphism $\widehat{\mathcal{I}}_{B} \rightarrow \widehat{\mathcal{L}}_{1}^{-1} \otimes \widehat{\mathcal{L}}_{2}$ for some $B \in$ $\Omega_{G}^{2}(M)$, and thus

$$
d_{G} B=\operatorname{curv}_{G}\left(\widehat{\mathcal{L}}_{2}\right)-\operatorname{curv}_{G}\left(\widehat{\mathcal{L}}_{1}\right) \in \Omega_{G}^{3}(M) .
$$

The above discussion may be summarized by the following proposition, which is given by combining Propositions 3.15, 3.24, 3.26 and [Sti, Proposition 3.2].

Proposition 3.27. The following is a commutative diagram of abelian groups

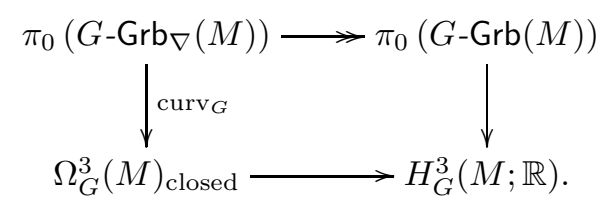

The upper horizontal map is surjective and determines the short exact sequence

$$
0 \rightarrow \frac{\Omega_{G}^{2}(M)}{\Omega_{G}^{2}(M)_{\mathbb{Z}}} \stackrel{\hat{\mathcal{I}}_{\bullet}}{\longrightarrow} \pi_{0}\left(G-\operatorname{Grb}_{\nabla}(M)\right) \rightarrow \pi_{0}(G-\operatorname{Grb}(M)) \rightarrow 0 .
$$


3.5. The induced simplicial gerbe. As observed in numerous sources (e.g. [Mei, MRSV, NS, Sti, TX]), an equivariant gerbe $\mathcal{L} \in G$-Grb $(M)$ naturally determines a gerbe $G \times \mathcal{L}$ on the simplicial manifold $G^{\bullet} \times M$. We write this as

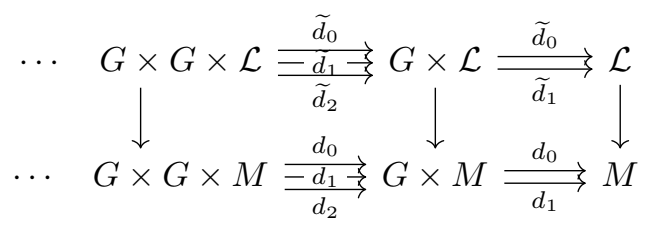

where $d_{i}$ denote the face maps, with $d_{0}(g, x)=x$ the source and $d_{1}(g, x)=g \cdot x$ the target. Explicitly, if $\mathcal{L}=(Y, L, \mu) \in G$ - $\operatorname{Grb}(M)$, the product gerbe $\left(G^{k} \times \mathcal{L}\right) \in \operatorname{Grb}\left(G^{k} \times M\right)$ is defined by $\left(G^{k} \times Y, G^{k} \times L\right.$, id $\left.\times \mu\right)$. The diagram (3.29) is then an abbreviated form of the following diagram.

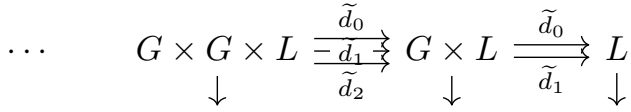

$$
\begin{aligned}
& \cdots \quad G \times G \times Y^{[2]} \rightrightarrows G \times Y^{[2]} \rightrightarrows Y^{[2]} \\
& \ldots \quad G \times G \times Y \Longrightarrow G \times Y \Longrightarrow \downarrow
\end{aligned}
$$

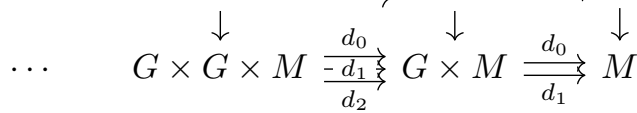

The maps $\widetilde{d}_{i}: G^{k+1} \times \mathcal{L} \rightarrow G^{k} \times \mathcal{L}$ determine a particularly strong form of gerbe isomorphism $d_{i}^{*}\left(G^{k} \times \mathcal{L}\right) \cong\left(G^{k+1} \times \mathcal{L}\right) \in \operatorname{Grb}\left(G^{k+1} \times M\right)$ where all structures are diffeomorphic; one does not need to first pass to another cover.

\section{Gerbes on the Quotient Stack}

We now give a different and more conceptual view of $G$-equivariant gerbe connections. As a simpler example, it is well-known that $G$-equivariant $S^{1}$-bundles on $M$ are equivalent to $S^{1}$-bundles on the Lie groupoid $G \times M \rightrightarrows M$, which are equivalent to $S^{1}$-bundles on the simplicial manifold $G^{\bullet} \times M$. When connections are introduced, the situation is more complicated. Given $P \in G$-Bun $S_{S^{1}}(M)$, a $G$-invariant connection on $P$ will not define a connection on the induced bundle over the Lie groupoid $G \times M \rightrightarrows M$ unless that connection was $\mathfrak{g}$-basic. However, when one realizes that $\mathcal{E} G \times_{G} M$ is the globally valid replacement of $M / / G$, it becomes natural to consider $\mathcal{E}_{\nabla} G \times_{G} M$, the version of the quotient stack that includes connections on the principal $G$-bundles.

We define $\operatorname{Grb}_{\nabla}\left(\mathcal{E}_{\nabla} G \times_{G} M\right):=\operatorname{Shv}_{\infty}\left(\mathcal{E}_{\nabla} G \times_{G} M, \mathcal{B}_{\nabla}^{2} S^{1}\right)$. In other words, an element $\widehat{\mathscr{L}} \in \operatorname{Grb}_{\nabla}\left(\mathcal{E}_{\nabla} G \times{ }_{G} M\right)$ assigns to every test manifold $X$ a functor

$$
\left(\mathcal{E}_{\nabla} G \times_{G} M\right)(X) \longrightarrow\left(\mathcal{B}_{\nabla}^{2} S^{1}\right)(X)=\operatorname{Grb}_{\nabla}(X),
$$

and it does so in a natural way compatible with pullback maps. Explicitly, a map of $G$-bundles $\varphi$ gives an isomorphism between the two induced gerbes with connection, as indicated in the following diagram.

$$
\stackrel{\left(P^{\prime}, \varphi^{*} \Theta\right) \stackrel{\varphi}{\rightarrow}(P, \Theta) \stackrel{f}{\rightarrow} M}{\downarrow_{X^{\prime}} \stackrel{\bar{\varphi}}{\longrightarrow} \underset{X}{\downarrow}} \mapsto \widehat{\mathscr{L}}\left(P^{\prime}, \varphi^{*} \Theta, f \circ \varphi\right) \stackrel{\widehat{\mathscr{L}}(\varphi)}{\longrightarrow} \bar{\varphi}^{*} \widehat{\mathscr{L}}(P, \Theta, f) \in \operatorname{Grb}_{\nabla}\left(X^{\prime}\right)
$$


We similarly define $\operatorname{Grb}\left(\mathcal{E} G \times_{G} M\right):=\operatorname{Shv}_{\infty}\left(\mathcal{E} G \times_{G} M, \mathcal{B}^{2} S^{1}\right)$. Fortunately, isomorphism classes of such gerbes may be classified via existing results and general theory.

Proposition 4.2. There are natural isomorphisms of abelian groups

$$
\begin{aligned}
& \pi_{0}\left(\operatorname{Grb}\left(\mathcal{E} G \times_{G} M\right)\right) \stackrel{\cong}{\rightrightarrows} H_{G}^{3}(M ; \mathbb{Z}), \\
& \pi_{0}\left(\operatorname{Grb}_{\nabla}\left(\mathcal{E}_{\nabla} G \times_{G} M\right)\right) \stackrel{\cong}{\rightarrow} \widehat{H}_{G}^{3}(M ; \mathbb{Z}) .
\end{aligned}
$$

Proof. The second isomorphism was already explained in Section 2.3. The Deligne complex gives a model for the 2-category of gerbes with connections, and hence there is an equivalence of sheaves

$$
\mathcal{B}_{\nabla}^{2} S^{1} \simeq \mathbb{L}\left(\Gamma\left(S^{1} \stackrel{d}{\rightarrow} \Omega^{1} \stackrel{d}{\rightarrow} \Omega^{2}\right)\right) \simeq \widehat{\mathcal{K}}(\mathbb{Z}, 3),
$$

which induces a natural isomorphism

$$
\pi_{0} \operatorname{Shv}_{\infty}\left(\mathcal{E}_{\nabla} G \times_{G} M, \mathcal{B}_{\nabla}^{2} S^{1}\right) \cong \pi_{0} \operatorname{Shv}_{\infty}\left(\mathcal{E}_{\nabla} G \times_{G} M, \widehat{\mathcal{K}}(\mathbb{Z}, 3)\right)=: \widehat{H}_{G}^{3}(M) .
$$

To see the corresponding result without connections, we know that the sheaf of bundle gerbes $\mathcal{B}^{2} S^{1}$ is equivalent to $\mathbb{L}\left(\Gamma\left(S^{1} \rightarrow 0 \rightarrow 0\right)\right)$. So, for any sheaf $\mathcal{M} \in \mathrm{Shv}_{\infty}$ we have

$$
\pi_{0} \operatorname{Grb}(\mathcal{M}):=\pi_{0} \operatorname{Shv}_{\infty}\left(\mathcal{M}, \mathcal{B}^{2} S^{1}\right) \cong H_{\text {Shv }}^{2}\left(\mathcal{M} ; S^{1}\right) .
$$

The short exact sequence of abelian Lie groups $0 \rightarrow \mathbb{Z} \hookrightarrow \mathbb{R} \rightarrow S^{1} \rightarrow 0$ induces a long exact sequence

$$
\cdots \rightarrow H_{\text {Shv }}^{2}\left(\mathcal{M} ; \Omega^{0}\right) \rightarrow H_{\text {Shv }}^{2}\left(\mathcal{M} ; S^{1}\right) \rightarrow H_{\text {Shv }}^{3}(\mathcal{M} ; \mathbb{Z}) \rightarrow H_{\text {Shv }}^{3}\left(\mathcal{M} ; \Omega^{0}\right) \rightarrow \cdots
$$

Here we write $\Omega^{0}$ to indicate that $\mathbb{R}$ is the sheaf of smooth real-valued functions, as opposed to the sheaf $\mathbb{R}^{\delta}$ of locally constant functions. By Lemma 4.3 below, which uses that $M / / G$ is a proper Lie groupoid, the first and last terms in the above sequence vanish and give an isomorphism

$$
0 \rightarrow H_{\text {Shv }}^{2}\left(\mathcal{E} G \times_{G} M, S^{1}\right) \cong H_{\text {Shv }}^{3}\left(\mathcal{E} G \times_{G} M, \mathbb{Z}\right) \rightarrow 0 .
$$

Finally, the sheaf $\mathbb{Z}$ is discrete, and hence

$$
H_{\text {Shv }}^{3}\left(\mathcal{E} G \times_{G} M ; \mathbb{Z}\right) \cong \pi_{0} \operatorname{Top}\left(E G \times_{G} M ; K(\mathbb{Z}, 3)\right)=H_{G}^{3}(M ; \mathbb{Z}),
$$

as explained in (2.20).

The following lemma, used in the proof of Proposition 4.2, is a special case of one of the main theorems from $[\mathrm{AC}]$, though their language looks different than ours. For that reason, we prove why our desired result follows from the theorem of Abad-Crainic.

Lemma 4.3 (Corollary 4.2 of $[\mathrm{AC}]$ ). Suppose that $G$ is a compact Lie group. If $n>0$, then $H_{\text {Shv }}^{n}\left(\mathcal{E} G \times_{G} M, \Omega^{0}\right)=0$.

Proof. If $G$ is compact, then the action of $G$ on $M$ is proper and $G \times M \rightrightarrows M$ is a proper Lie groupoid. Thus, Corollary 4.2 of $[\mathrm{AC}]$ implies the (co)homology of

$$
\Omega^{0}(M) \stackrel{\delta}{\rightarrow} \Omega^{0}(G \times M) \stackrel{\delta}{\rightarrow} \Omega^{0}\left(G^{2} \times M\right) \stackrel{\delta}{\rightarrow} \cdots \stackrel{\delta}{\rightarrow} \Omega^{0}\left(G^{n} \times M\right) \stackrel{\delta}{\rightarrow} \cdots
$$

vanishes at all terms where $n>0$.

Our definition of sheaf cohomology in (2.19) states that

$$
H_{\text {Shv }}^{n}\left(\mathcal{E} G \times_{G} M, \Omega^{0}\right):=\pi_{0} \operatorname{Shv}_{\infty}\left(\mathcal{E} G \times_{G} M, \mathbb{L}\left(\Gamma\left(\Omega^{0}[-n]\right)\right)\right) .
$$


One can actually calculate this by the following:

$$
\begin{aligned}
\operatorname{Shv}_{\infty} & \left(\mathcal{E} G \times_{G} M, \mathbb{L}\left(\Gamma\left(\Omega^{0}[-n]\right)\right)\right) \simeq \operatorname{Shv}_{\infty}\left(\mathbb{L}(M / / G), \mathbb{L}\left(\Gamma\left(\Omega^{0}[-n]\right)\right)\right) \\
& \simeq \operatorname{Pre}_{\infty}\left(M / / G, \mathbb{L}\left(\Gamma\left(\Omega^{0}[-n]\right)\right)\right) \simeq \operatorname{Pre}_{\infty}\left(M / / G, \Gamma\left(\Omega^{0}[-n]\right)\right) \\
& \simeq \operatorname{Pre}_{\infty}\left(\underset{\Delta^{\mathrm{op}}}{\operatorname{hocolim}} G^{\bullet} \times M, \Gamma\left(\Omega^{0}[-n]\right)\right) \simeq{\operatorname{\Delta ^{\mathrm {op}}}}_{\operatorname{Pre}} \operatorname{Pr}_{\infty}\left(G^{\bullet} \times M, \Gamma\left(\Omega^{0}[-n]\right)\right) \\
& \simeq \operatorname{holim}_{\Delta^{\mathrm{op}}} \Gamma\left(\Omega^{0}\left(G^{\bullet} \times M\right)[-n]\right) \simeq \Gamma\left(\cdots \stackrel{\delta}{\rightarrow} \Omega^{0}\left(G^{n-1} \times M\right) \stackrel{\delta}{\rightarrow} \Omega^{0}\left(G^{n} \times M\right)_{\delta \text {-cl }}\right) .
\end{aligned}
$$

The above string of equivalences is justified by the following. The first equivalence follows from $\mathbb{L}(M / / G) \simeq \mathcal{E} G \times_{G} M$, as noted in Remark 2.8. The second is because sheafification is

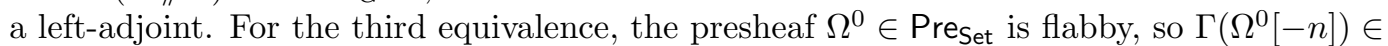
Pre $_{\infty}$ satisfies the descent property of $\operatorname{Shv}_{\infty}$ and $\left.\mathbb{L}\left(\Gamma\left(\Omega^{0}[-n]\right)\right) \simeq \Gamma\left(\Omega^{0}[-n]\right)\right)$. To see the fourth, for any simplicial set $B$ there is a natural equivalence hocolim ${ }_{\Delta}$ op $N(S) \simeq B$, where we use the composition $\Delta^{\text {op }} \stackrel{S}{\rightarrow}$ Set $\stackrel{N}{\rightarrow}$ sSet to consider $N(S)$ as a simplicial object in simplicial sets. The fifth is a general property of colimits, and the sixth is Yoneda's lemma. The seventh is a simplification of the general principal that in chain complexes, homotopy limits over a simplicial diagram may be calculated via the total double complex; see [Dug, Section 19.8] or [BSS, Appendix B.1].

Finally, $\pi_{0}$ of the resulting simplicial set is simply $\Omega^{0}\left(G^{n} \times M\right)_{\delta \text {-cl }} / \delta\left(\Omega^{0}\left(G^{n-1} \times M\right)\right)$, which is precisely what is proven to vanish by Abad-Crainic.

4.1. The functor between the two models. Our goal is now to relate these two models of equivariant gerbe connections, given by $G-\operatorname{Grb}_{\nabla}(M)$ and $\operatorname{Grb}_{\nabla}\left(\mathcal{E}_{\nabla} G \times_{G} M\right)$, in a way analogous to (2.17). The following proposition will be the key to doing so, and it generalizes the construction of (2.16): given $(P, \Theta) \in \operatorname{Bun}_{G, \nabla}(X)$ and $(L, \nabla) \in G$-Bun $S^{1}, \nabla(P)$, there is a canonical way to modify $\nabla$ so that it becomes $G$-basic and descends to the quotient $L / G$.

\section{Proposition 4.4.}

(1) A principal $G$-bundle $P \in \operatorname{Bun}_{G}(X)$ determines a natural functor

$$
\begin{aligned}
G-\operatorname{Grb}(P) & \stackrel{/ G}{\longrightarrow} \operatorname{Grb}(X) \\
\mathcal{L} & \longmapsto \mathcal{L} / G .
\end{aligned}
$$

(2) A principal $G$-bundle with connection $(P, \Theta) \in \operatorname{Bun}_{G, \nabla}(X)$ determines a natural functor

$$
G-\operatorname{Grb}_{\nabla}(P) \stackrel{\Theta^{*}}{\longrightarrow} \operatorname{Grb}_{\nabla}(X),
$$

and the curvature satisfies

$$
\operatorname{curv}\left(\Theta^{*} \widehat{\mathcal{L}}\right)=\Theta^{*}\left(\operatorname{curv}_{G}(\widehat{\mathcal{L}})\right) \in \Omega^{3}(X)
$$

$$
\text { under } \Omega_{G}^{3}(P) \stackrel{\Theta^{*}}{\longrightarrow} \Omega^{3}(X) \text {. }
$$

Proof. We first do the topological case, which is straightforward. Let $\mathcal{L}=(Y, L, \mu) \in$ $G$-Grb $(P)$. Since $G$ acts freely on $P$, it also acts freely on $Y, Y^{[2]}$, and $L$. Therefore, we have the following commutative diagram,

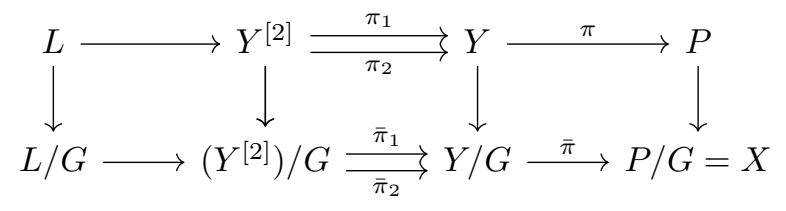


where each vertical map is a principal $G$-bundle.

Because $G$ acts freely on $P$ and $Y$, there is a natural diffeomorphism

$$
\left(Y^{[2]}\right) / G=\left(Y \times_{P} Y\right) / G \cong(Y / G) \times_{P / G}(Y / G)=(Y / G)^{[2]}
$$

To see this, observe that the projection $Y \times_{P} Y \rightarrow(Y / G) \times_{P / G}(Y / G)$ fits into the commutative diagram

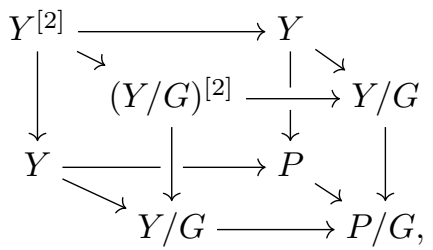

where the front and rear faces are pullbacks of surjective submersions, and the other four faces are pullback squares of principal $G$-bundles. This gives $Y^{[2]} \rightarrow(Y / G)^{[2]}$ the structure of a principal $G$-bundle and produces the desired diffeomorphism (4.6).

The original bundle gerbe multiplication $\mu$ is $G$-equivariant and naturally descends to a map on the quotients

$$
\bar{\pi}_{12}^{*}(L / G) \otimes \bar{\pi}_{23}^{*}(L / G) \stackrel{\mu / G}{\longrightarrow} \bar{\pi}_{13}^{*}(L / G),
$$

where $\bar{\pi}_{i j}:(Y / G)^{[3]} \rightarrow(Y / G)^{[2]}$ denotes the induced map on the quotient manifolds. Therefore, the bottom row of (4.5) is the data of the bundle gerbe on $P / G=X$,

$$
\mathcal{L} / G:=(Y / G, L / G, \mu / G) \in \operatorname{Grb}(X) .
$$

Similarly, any $G$-equivariant 1-morphism $\mathcal{K}=(Z, K, \alpha)$ or 2-morphism $\mathcal{J}=(W, \beta)$ descends to an ordinary bundle gerbe 1-morphism $\mathcal{K} / G:=(Z / G, K / G, \alpha / G)$ or 2-morphism $\mathcal{J} / G:=$ $(W / G, \beta / G)$. This establishes the desired functor $G-\operatorname{Grb}(P) \rightarrow \operatorname{Grb}(P / G)$ when $G$ acts freely on $P$.

For the second part, assume $(P, \Theta) \in \operatorname{Bun}_{G, \nabla}(X)$. We define

$$
\Theta^{*} \widehat{\mathcal{L}}:=\left(Y / G, \Theta^{*}(L, \nabla), \Theta^{*} B, \mu / G\right) \in \operatorname{Grb}_{\nabla}(X),
$$

where $\Theta^{*}(L, \nabla) \in \operatorname{Bun}_{S^{1}, \nabla}\left((Y / G)^{[2]}\right)$ and $\Theta^{*} B \in \Omega^{2}(Y / G)$ are given by (2.14) and (2.10), respectively. The compatibility between $\Theta^{*} B$ and $\Theta^{*}(L, \nabla)$, along with the calculation of $\operatorname{curv}\left(\Theta^{*} \widehat{\mathcal{L}}\right)$, follows directly from the naturality of the Weil homomorphism. First,

$$
\operatorname{curv}\left(\Theta^{*} \nabla\right)=\Theta^{*}\left(\operatorname{curv}_{G}(\nabla)\right)=\Theta^{*}\left(\pi_{2}^{*} B-\pi_{1}^{*} B\right)=\bar{\pi}_{2}^{*}\left(\Theta^{*} B\right)-\bar{\pi}_{1}^{*}\left(\Theta^{*} B\right) \in \Omega^{2}\left((Y / G)^{[2]}\right) .
$$

Second, since

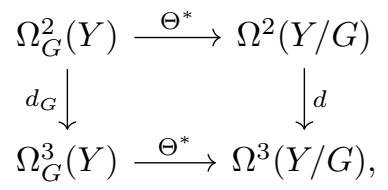

we immediately obtain

$$
\bar{\pi}^{*} \operatorname{curv}\left(\Theta^{*} \widehat{\mathcal{L}}\right)=d\left(\Theta^{*} B\right)=\Theta^{*} d_{G} B=\Theta^{*}\left(\pi^{*} \operatorname{curv}_{G}(\widehat{\mathcal{L}})\right)=\bar{\pi}^{*}\left(\Theta^{*} \operatorname{curv}_{G}(\widehat{\mathcal{L}})\right) .
$$

The map $\bar{\pi}^{*}$ is injective, therefore $\operatorname{curv}\left(\Theta^{*} \widehat{\mathcal{L}}\right)=\Theta^{*} \operatorname{curv}_{G}(\widehat{\mathcal{L}})$.

Similarly, any $G$-equivariant 1 -morphism with connection $\widehat{\mathcal{K}}=\left(Z, K, \nabla^{K}, \alpha\right)$ is mapped to $\Theta^{*} \widehat{\mathcal{K}}:=\left(Z / G, \Theta^{*}\left(K, \nabla^{K}\right), \alpha / G\right)$. For a 2-morphism, $\widehat{\mathcal{J}}$ contains no additional data past $\mathcal{J}$, it merely satisfies further requirements. 
There is now an obvious map

$$
\begin{aligned}
G-\operatorname{Grb}_{\nabla}(M) & \longrightarrow \operatorname{Grb}_{\nabla}\left(\mathcal{E}_{\nabla} G \times_{G} M\right) \\
\widehat{\mathcal{L}} & \longmapsto \widehat{\mathscr{L}},
\end{aligned}
$$

given by the following. For a moment, we use $\phi$ to denote the connection 1-form so as not to confuse between connection forms and covariant derivatives. If $\widehat{\mathcal{L}}=(Y, L, \phi, B, \mu)$, then for $(P, \Theta, f) \in\left(\mathcal{E}_{\nabla} G \times_{G} M\right)(X)$ use the composition

$$
G-\operatorname{Grb}_{\nabla}(M) \stackrel{f^{*}}{\longrightarrow} G-\mathrm{Grb}_{\nabla}(P) \stackrel{\Theta^{*}}{\longrightarrow} \operatorname{Grb}_{\nabla}(X)
$$

to define

$$
\widehat{\mathscr{L}}(P, \Theta, f):=\Theta^{*}\left(f^{*} \widehat{\mathcal{L}}\right) \in \operatorname{Grb}_{\nabla}(X) .
$$

Explicitly, this can be written as

$$
\widehat{\mathscr{L}}(P, \Theta, f)=\left(\left(f^{*} Y\right) / G,\left(f^{*} L\right) / G,\left(1-\iota_{\Theta}\right) f^{*} \phi, \Theta^{*}\left(f^{*} B\right),\left(f^{*} \mu\right) / G\right),
$$

as seen through the diagram

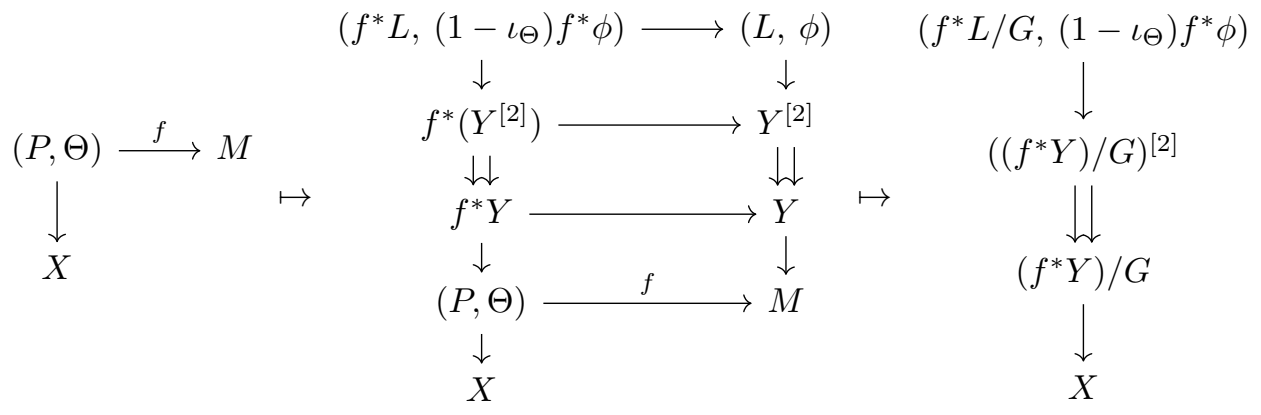

Similarly, we define

$$
\begin{aligned}
G-\operatorname{Grb}(M) & \longrightarrow \operatorname{Grb}\left(\mathcal{E} G \times{ }_{G} M\right) \\
\mathcal{L} & \longmapsto \mathscr{L}
\end{aligned}
$$

by $\mathscr{L}(P, f):=\left(f^{*} \mathcal{L}\right) / G \in \operatorname{Grb}(X)$ for $(P, f) \in\left(\mathcal{E} G \times_{G} M\right)(X)$.

Using the functors (4.7) or (4.9), along with Proposition 4.2, we now define the equivariant Dixmier-Douady class $\operatorname{DD}_{G}(\widehat{\mathcal{L}})=\operatorname{DD}_{G}(\mathcal{L}) \in H_{G}^{3}(M ; \mathbb{Z})$ as the image of $\widehat{\mathcal{L}}$ or $\mathcal{L}$ in the composition

$$
\pi_{0}\left(G-\operatorname{Grb}_{\nabla}(M)\right) \rightarrow \pi_{0}(G-\operatorname{Grb}(M)) \rightarrow \pi_{0}\left(\operatorname{Grb}\left(\mathcal{E} G \times_{G} M\right)\right) \stackrel{\cong}{\rightarrow} H_{G}^{3}(M ; \mathbb{Z}) .
$$

Our present goal is to show the second map in (4.10) is an isomorphism. Fortunately, $\mathrm{Tu}-\mathrm{Xu}$ already proved that the homomorphism $\pi_{0}(G$-Grb $(M)) \rightarrow H_{G}^{3}(M ; \mathbb{Z})$ is surjective. In [TX, Theorem 2.8, Corollary 2.10], they show that for any class $\xi \in H_{G}^{3}(M ; \mathbb{Z})$, there exists some $\mathcal{L} \in G$ - $\operatorname{Grb}(M)$ with $\mathrm{DD}_{G}(\mathcal{L})=\xi$.

It therefore remains for us to show the homomorphism $\pi_{0}(G$-Grb $(M)) \rightarrow H_{G}^{3}(M ; \mathbb{Z})$ is injective, a fact that was previously noted but not proven in Remark 5.8 of [Sti]. Our proof relies on better understanding the behavior of $\mathcal{L} \mapsto \mathscr{L}$ and using this to show that $\pi_{0}(G-\operatorname{Grb}(M)) \rightarrow \pi_{0}\left(\operatorname{Grb}\left(\mathcal{E} G \times_{G} M\right)\right)$ is an injection.

Proposition 4.11. Let $\mathcal{L} \in G-\operatorname{Grb}(M)$. The image of $\mathcal{L}$ in the composition

$$
G-\operatorname{Grb}(M) \rightarrow \operatorname{Grb}\left(\mathcal{E} G \times_{G} M\right) \rightarrow \operatorname{Grb}(M / / G)
$$


is isomorphic to $G^{\bullet} \times \mathcal{L} \in \infty-\mathrm{Gpd}$, as defined in (3.29).

Proof. The simplicial manifold $G^{\bullet} \times M=M / / G=N(G \times M \rightrightarrows M)$ has a natural map to $\mathcal{E} G \times{ }_{G} M$ induced by the trivial $G$-bundle. First, consider $M \rightarrow \mathcal{E} G \times_{G} M$ defined by

$$
\begin{aligned}
& G \times M \stackrel{d_{1}}{\longrightarrow} M \\
& d_{0} \prod_{i}^{\uparrow} s_{0} \\
& \text { M, }
\end{aligned}
$$

where we view $G \times M$ as a trivial principal (left) $G$-bundle over $M$, and $d_{1}: G \times M \rightarrow M$ as the $G$-equivariant map $(g, m) \mapsto g \cdot m$; here $G$ acts on $G \times M$ by left multiplication on $G$ only. The bundle also has a natural section of $d_{0}$ given by the degeneracy map $s_{0}: M \rightarrow G \times M$, since $d_{0} s_{0}=\mathrm{id}$. Thus, the value of $\mathscr{L}$ on $d_{1}$ is given by

$$
\mathscr{L}\left(G \times M, d_{1}\right)=\left(d_{1}^{*} \mathcal{L}\right) / G \cong s_{0}^{*}\left(d_{1}^{*} \mathcal{L}\right) \cong\left(d_{1} \circ s_{0}\right)^{*} \mathcal{L} \cong \mathcal{L} \in \operatorname{Grb}(M) .
$$

The remaining components of the natural map $M / / G \rightarrow \mathcal{E} G \times_{G} M$ can be seen through the following.

$$
\begin{aligned}
& \text {.. } G \times G \times G \times M \underset{d_{3}}{\stackrel{d_{1}}{-\frac{d_{2}}{\rightrightarrows}}} G \times G \times M \underset{d_{2}}{\stackrel{d_{1}}{\longrightarrow}} G \times M \stackrel{d_{1}}{\longrightarrow} M
\end{aligned}
$$

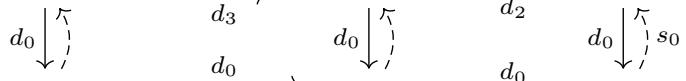

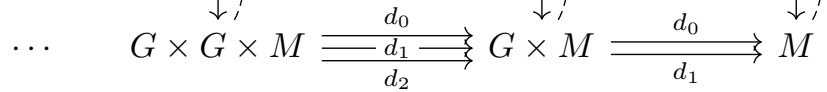

This diagram commutes because of the simplicial identities $d_{0} d_{j}=d_{j-1} d_{0}$ for $j>0$, and hence it forms a simplicial $G$-bundle on $G^{\bullet} \times M$ with an equivariant map to $M$.

To compute $\mathscr{L}$ when evaluated on $M / / G$, note that we have a natural isomorphism between the pullback of $\mathcal{L}$ in (4.12) and the following diagram.

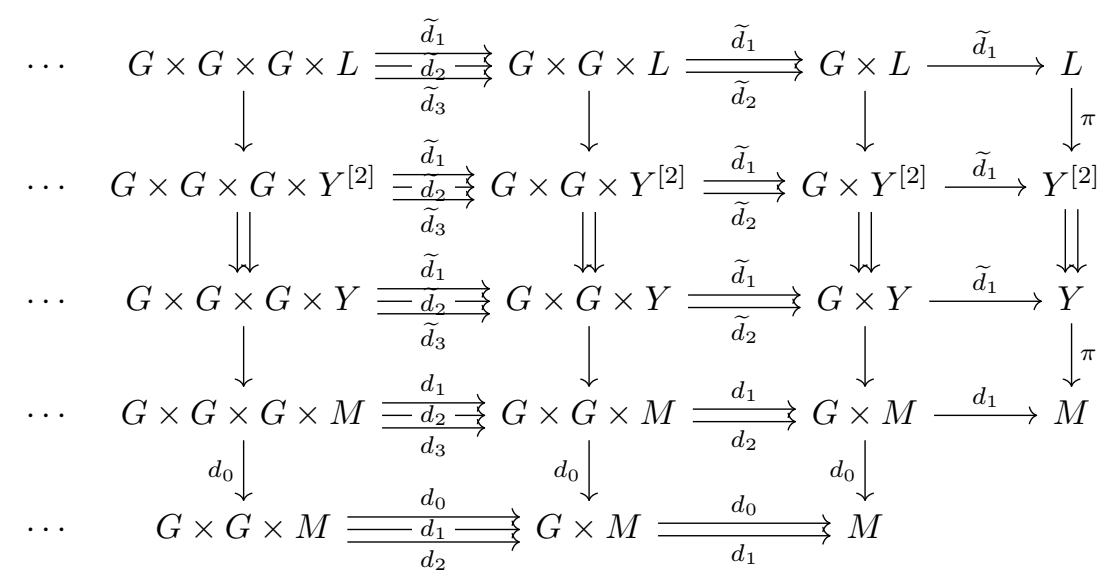

Furthermore, the section $s_{0}$ pulls back along each map in the following way: $d_{i}^{*} s_{0}=s_{0}$ for $i \geq 1$ because $d_{i+1} s_{0}=s_{0} d_{i}$. This gives a natural identification $\widetilde{d}_{i+1} / G \cong \widetilde{d}_{i}$ for $i \geq 1$. For $i=0$, we have that $\left(d_{0}^{*} s_{0}\right)\left(g_{1}, g_{2}, \ldots g_{k}, x\right)=\left(g_{1}^{-1}, g_{1}, g_{2}, \ldots g_{k}, x\right)$. Therefore,

$$
\left(\tilde{d}_{1} / G\right)\left(g_{1}, \ldots, g_{k}, x\right)=d_{0} \widetilde{d}_{1}\left(g_{1}^{-1}, g_{1}, \ldots, g_{k}, x\right)=d_{0}\left(1, g_{2}, \ldots, g_{k}, x\right)=\left(g_{2}, \ldots g_{k}, x\right),
$$

giving $\left(\widetilde{d}_{1}\right) / G=d_{0}$. Hence, when one quotients the above diagram by $G$, the result is a diagram that is naturally isomorphic to the diagram (3.30) defining $G^{\bullet} \times \mathcal{L}$. 


\subsection{Proof of equivalence.}

Proposition 4.13. The group homomorphism $\pi_{0}(G-\operatorname{Grb}(M)) \rightarrow \pi_{0}\left(\operatorname{Grb}\left(\mathcal{E} G \times_{G} M\right)\right)$ is an injection.

Proof. Consider an arbitrary $\mathcal{L}=(Y, L, \mu) \in G$ - $\operatorname{Grb}(M)$ with induced $\mathscr{L} \in \operatorname{Grb}\left(\mathcal{E} G \times{ }_{G} M\right)$. Suppose there is an isomorphism $\mathscr{K}: \mathscr{I} \rightarrow \mathscr{L}$, where $\mathscr{I}$ is the image of the trivial gerbe $\mathcal{I}$. Evaluating $\mathscr{K}$ on the natural map $M \rightarrow \mathcal{E} G \times{ }_{G} M$, described in Proposition 4.11, gives an ordinary non-equivariant trivialization

$$
\mathcal{I} \cong \mathscr{I}\left(G \times M, d_{1}\right) \underset{\mathcal{K}:=\mathscr{K}\left(G \times M, d_{1}\right)}{\cong} \mathscr{L}\left(G \times M, d_{1}\right) \cong \mathcal{L}
$$

in $\operatorname{Grb}(M)$. As discussed in Section 3.4, we may assume $\mathcal{K}$ is given by $K \in \operatorname{Bun}_{S^{1}}(Y)$ with an isomorphism $\alpha: \delta K \rightarrow L$, which is a priori non-equivariant. But, $\mathcal{K}$ extends to a trivialization on $\mathscr{L}\left(G^{\bullet} \times M\right) \cong G^{\bullet} \times \mathcal{L}$. Hence, the bundle $K \in \operatorname{Bun}_{S^{1}}(Y)$ extends to a bundle on $\operatorname{Bun}_{S^{1}}(Y / / G)$. The well-known equivalence $\operatorname{Bun}_{S^{1}}(Y / / G) \cong G$-Bun $S_{S^{1}}(Y)$, proven in [Red2, Theorem 4.3], therefore implies that $K \in G$-Bun $S_{S^{1}}(Y)$. Since the isomorphism $\alpha$ also extends to $G^{\bullet} \times \mathcal{L}$, this implies that $\alpha$ is equivariant with respect to the induced induced $G$-structure on $K$. Therefore, our morphism $\mathcal{K}$ is $G$-equivariant and provides a trivialization of $\mathcal{L} \in G-\operatorname{Grb}(M)$.

Proposition 4.14. The equivariant Dixmier-Douady class gives a natural isomorphism of abelian groups

$$
\mathrm{DD}_{G}: \pi_{0}(G-\operatorname{Grb}(M)) \stackrel{\cong}{\rightrightarrows} \pi_{0}\left(\operatorname{Grb}\left(\mathcal{E} G \times_{G} M\right)\right) \stackrel{\cong}{\rightrightarrows} H_{G}^{3}(M ; \mathbb{Z}) .
$$

Proof. Proposition 4.2 says the second map is an isomorphism. Theorem 2.8 of [TX] says the composition is surjective, and hence the first map is a surjection. Proposition 4.13 says the first map is an injection. Hence, the first map must also be an isomorphism.

Theorem 4.15. Let $G$ be a compact Lie group. For $M \in G$-Man, there is a natural isomorphism of abelian groups

$$
\pi_{0}\left(G-\operatorname{Grb}_{\nabla}(M)\right) \cong \widehat{H}_{G}^{3}(M)
$$

between \{equivalence classes of $G$-equivariant gerbes with connection on $M$ \} and the third differential equivariant cohomology group of $M$. Furthermore, the character diagram

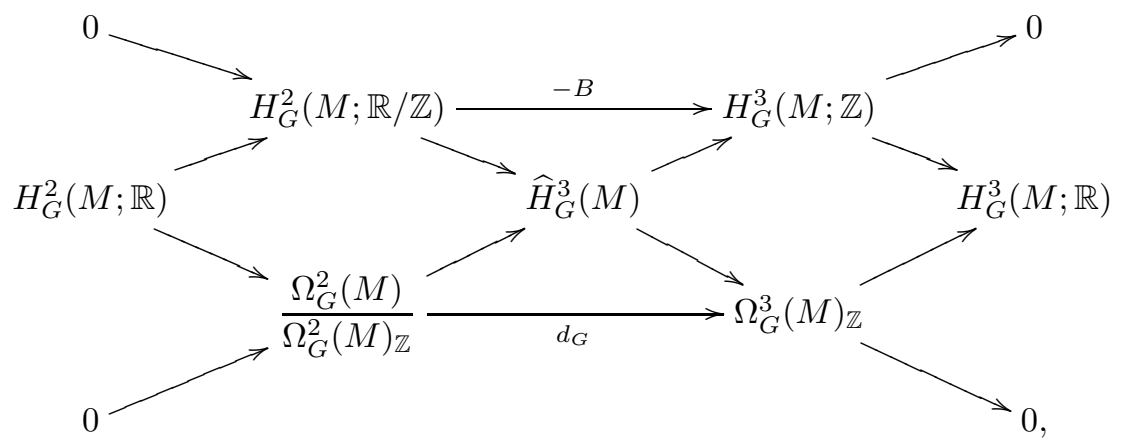


in which the two diagonals are both short exact sequences, is isomorphic to the following character diagram.

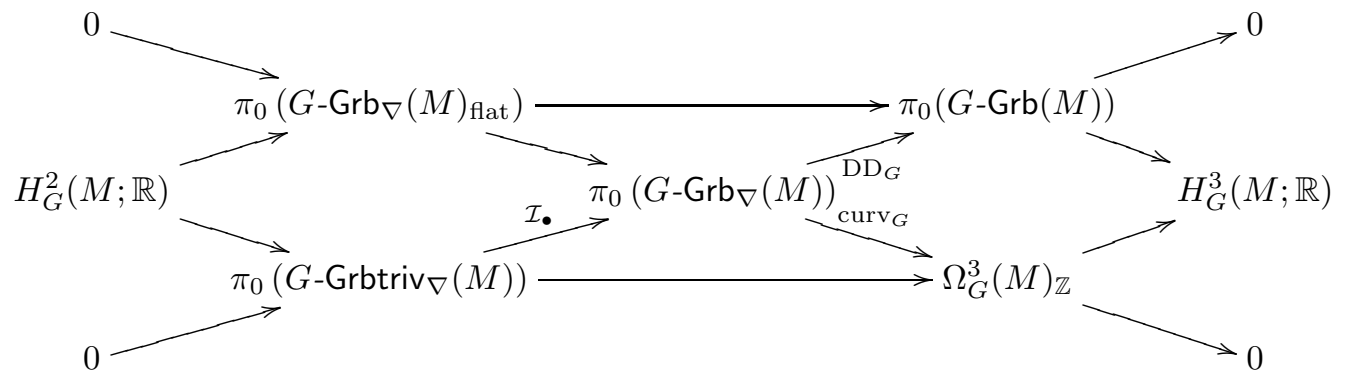

Proof. The theorem follows from Proposition 3.27, Proposition 4.14, and the five lemma. Consider the commutative diagram

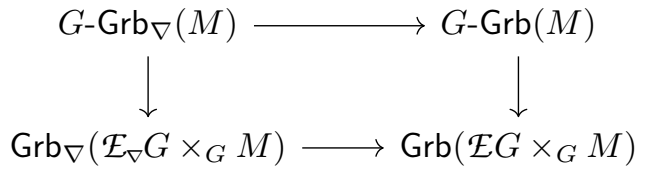

where the vertical maps were defined in (4.7) and (4.9), and the horizontal maps are given by forgetting connection data. Taking isomorphism classes gives a homomorphism of short exact sequences

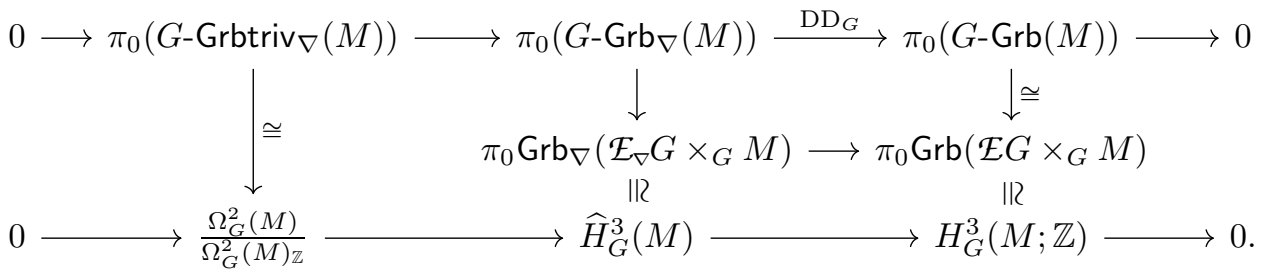

The upper sequence was proven to be exact in Proposition 3.27; the lower two vertical isomorphisms were established in Proposition 4.2; the upper-right vertical isomorphism was proven in Proposition 4.14; and the lower short exact sequence is (2.22). Therefore, the five lemma implies the homomorphism $\pi_{0}\left(G-G_{r b}(M)\right) \rightarrow \widehat{H}_{G}^{3}(M)$ is an isomorphism. This also shows that the positively-sloped diagonals in the two character diagrams are isomorphic.

It remains to verify that the two curvature maps are compatible, in the sense that the diagram

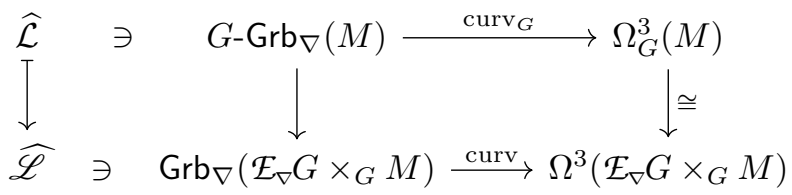

commutes. To determine $\operatorname{curv}(\widehat{\mathscr{L}}) \in \Omega^{3}\left(\mathcal{E}_{\nabla} G \times_{G} M\right)$, it suffices to evaluate on all possible $(P, \Theta, f): X \rightarrow \mathcal{E}_{\nabla} G \times_{G} M$. Using the definition of $\widehat{\mathscr{L}}$ in (4.8), along with Proposition 4.4, we see that

$$
\operatorname{curv}(\widehat{\mathscr{L}}(P, \Theta, f))=\operatorname{curv}\left(\Theta^{*}\left(f^{*} \widehat{\mathcal{L}}\right)\right)=\Theta^{*}\left(\operatorname{curv}_{G}\left(f^{*} \widehat{\mathcal{L}}\right)\right)=\Theta^{*}\left(f^{*}\left(\operatorname{curv}_{G}(\widehat{\mathcal{L}})\right) \in \Omega^{3}(X) .\right.
$$

This is precisely the image of $\operatorname{curv}_{G}(\widehat{\mathcal{L}})$ in $\Omega^{3}\left(\mathcal{E}_{\nabla} G \times_{G} M\right)$ when evaluated on $(P, \Theta, f)$, as defined in (2.12). 
Finally, note that $G-\mathrm{Grb}_{\nabla}(M)_{\text {flat }}$ is defined as the full subgroupoid of $G$-Grb $\nabla(M)$ whose objects have curvature 0 . Thus, the sequence

$$
0 \rightarrow \pi_{0}\left(G-\operatorname{Grb}_{\nabla}(M)\right)_{\text {flat }} \rightarrow \pi_{0}\left(G-\operatorname{Grb}_{\nabla}(M)\right) \stackrel{\operatorname{curv}_{G}}{\longrightarrow} \Omega^{3}(M)_{\mathbb{Z}} \rightarrow 0
$$

is exact, and we use the existing short exact sequence (2.21) to define the isomorphism

$$
H_{G}^{2}(M ; \mathbb{R} / \mathbb{Z}) \stackrel{\cong}{\rightrightarrows} \pi_{0}\left(G-\operatorname{Grb}_{\nabla}(M)_{\text {flat }}\right) .
$$

Therefore, the isomorphism $\pi_{0}\left(G-G_{r b}(M)\right) \stackrel{\cong}{\rightrightarrows} \widehat{H}_{G}^{3}(M)$ induces an isomorphism between the two character diagrams.

Theorem 4.16. Let $G$ be a compact Lie group and $M \in G$-Man. The natural functors

$$
\begin{aligned}
G-\operatorname{Grb}(M) & \stackrel{\simeq}{\longrightarrow} \operatorname{Grb}\left(\mathcal{E} G \times_{G} M\right), \\
G-\operatorname{Grb}_{\nabla}(M) & \stackrel{\simeq}{\longrightarrow} \operatorname{Grb}_{\nabla}\left(\mathcal{E}_{\nabla} G \times_{G} M\right),
\end{aligned}
$$

defined in (4.7) and (4.9), are both equivalences of 2-groupoids in $\infty$-Gpd.

Proof. Proposition 4.14 and Theorem 4.15 show that both functors are bijections on isomorphism classes of objects. As explained in Section 2.1, we must now show that both functors induce equivalences between all automorphism groupoids. As explained in (3.17), the Picard structures naturally identify automorphisms of a general object with automorphisms for the trivial object, which greatly simplifies the remaining task.

Corollary 3.25 gives an equivalence $\operatorname{Aut}(\mathcal{L}) \simeq G$-Bun $S^{1}(M)$ for any $\mathcal{L} \in G-\operatorname{Grb}(M)$. Similarly, the automorphism groupoid of any $\mathscr{L} \in \operatorname{Grb}\left(\mathcal{E} G \times_{G} M\right)$ is equivalent to

$\operatorname{Aut}(\mathscr{I}) \simeq \operatorname{Shv}_{\infty}\left(\mathcal{E} G \times_{G} M, \mathbb{L}\left(\Gamma\left(S^{1} \rightarrow 0\right)\right)\right) \simeq \operatorname{Shv}_{\infty}\left(\mathcal{E} G \times_{G} M, \mathcal{B} S^{1}\right)=\operatorname{Bun}_{S^{1}}\left(\mathcal{E} G \times_{G} M\right)$.

It is a well-known result that the induced functor $G$-Bun $\operatorname{Bun}^{1}(M) \stackrel{\cong}{\rightrightarrows} \operatorname{Bun}_{S^{1}}\left(\mathcal{E} G \times \times_{G} M\right)$ is an equivalence of categories $[\operatorname{Red} 2$, Theorem 4.3]. Therefore, we may conclude that $G-\operatorname{Grb}(M) \stackrel{\simeq}{\rightarrow} \operatorname{Grb}\left(\mathcal{E} G \times_{G} M\right)$ is an equivalence.

Corollary 3.25 also shows $\operatorname{Aut}(\widehat{\mathcal{L}}) \simeq G$-Bun $S_{S^{1}, \nabla}(M)_{\text {flat }}$ for $\widehat{\mathcal{L}} \in G-\operatorname{Grb}_{\nabla}(M)$. For $\widehat{\mathscr{L}} \in$ $\operatorname{Grb}_{\nabla}\left(\mathcal{E}_{\nabla} G \times_{G} M\right)$,

$$
\operatorname{Aut}(\widehat{\mathscr{L}}) \cong \operatorname{Aut}\left(\widehat{\mathscr{I})} \simeq \operatorname{Shv}_{\infty}\left(\mathcal{E}_{\nabla} G \times_{G} M, \mathbb{L}\left(\Gamma\left(S^{1} \rightarrow \Omega_{\mathrm{cl}}^{1}\right)\right)\right),\right.
$$

which is the subgroupoid of $\operatorname{Shv}_{\infty}\left(\mathcal{E}_{\nabla} G \times_{G} M, \mathbb{L}\left(\Gamma\left(S^{1} \rightarrow \Omega^{1}\right)\right)\right) \simeq \operatorname{Shv}_{\infty}\left(\mathcal{E}_{\nabla} G \times_{G} M, \mathcal{B}_{\nabla} S^{1}\right)$ consisting of objects whose curvature is $0 \in \Omega^{2}\left(\mathcal{E}_{\nabla} G \times_{G} M\right)$. In other words, $\operatorname{Aut}(\widehat{\mathscr{L}}) \simeq$ $\operatorname{Bun}_{S^{1}, \nabla}\left(\mathcal{E}_{\nabla} G \times_{G} M\right)_{\text {flat }}$. The equivalence $G-\operatorname{Bun}_{S^{1}, \nabla}(M) \stackrel{\cong}{\rightrightarrows} \operatorname{Bun}_{S^{1}, \nabla}\left(\mathcal{E}_{\nabla} G \times_{G} M\right)$ from [Red2, Theorem 4.3], when restricted to the subgroupoids of flat connections, implies

$$
\operatorname{Aut}(\widehat{\mathcal{L}}) \simeq G-\operatorname{Bun}_{S^{1}, \nabla}(M)_{\text {flat }} \cong \operatorname{Bun}_{S^{1}, \nabla}\left(\mathcal{E}_{\nabla} G \times_{G} M\right)_{\text {flat }} \simeq \operatorname{Aut}(\widehat{\mathscr{L}})
$$

\section{Applications}

The results from the previous section have a few immediate consequences that we briefly explain. 
5.1. Equivariant holonomy. One important general principle is the following: Any natural construction involving gerbes has an equivariant generalization, because equivariant gerbes pull back to ordinary gerbes. More precisely, $\widehat{\mathcal{L}} \in G$-Grb $\nabla(M)$ determines $\widehat{\mathscr{L}} \in$ $\operatorname{Grb}_{\nabla}\left(\mathcal{E}_{\nabla} G \times_{G} M\right)$, which pulls back along any $(P, \Theta, f): X \rightarrow \mathcal{E}_{\nabla} G \times_{G} M$ to an ordinary gerbe with connection $\widehat{\mathscr{L}}(P, \Theta, f) \in \mathrm{Grb}_{\nabla}(X)$, as indicated by

$$
X \stackrel{(P, \Theta, f)}{\longrightarrow} \mathcal{E}_{\nabla} G \times_{G} M \stackrel{\widehat{\mathscr{L}}}{\longrightarrow} \mathcal{B}_{\nabla}^{2} S^{1} .
$$

A specific instance of this principle is surface holonomy for gerbes with connection. Holonomy for ordinary gerbe connections is defined in a number of ways, and it classifies gerbe connections up to isomorphism; see [BTW, GR, FNSW, MP] for various treatments. If $\Sigma^{2}$ is a closed oriented surface, then any gerbe connection on $\Sigma^{2}$ is isomorphic to $\widehat{\mathcal{I}}_{B}$ for some $B \in \Omega^{2}\left(\Sigma^{2}\right)$. The holonomy is defined by

$$
\operatorname{Hol}_{\widehat{\mathcal{I}}_{B}}:=\int_{\Sigma^{2}} B \bmod \mathbb{Z} \in \mathbb{R} / \mathbb{Z}
$$

where $S^{1}$ continues to be identified with $\mathbb{R} / \mathbb{Z}$ to avoid factors of $2 \pi i$. For general $\widehat{\mathcal{L}} \in$ $\operatorname{Grb}_{\nabla}(M)$, the holonomy $\operatorname{Hol}_{\widehat{\mathcal{L}}}(f)$ along $f: \Sigma^{2} \rightarrow M$ is simply Hol $\widehat{\mathcal{I}}_{B}$, where $f^{*} \widehat{\mathcal{L}} \cong \widehat{\mathcal{I}}_{B}$.

This existing holonomy construction immediately defines a version for $G$-equivariant gerbe connections. Suppose that $M \in G$-Man and $\widehat{\mathcal{L}} \in G$-Grb $\operatorname{Gr}_{\nabla}(M)$. Given $(P, \Theta) \in$ $\operatorname{Bun}_{G, \nabla}\left(\Sigma^{2}\right)$ and a $G$-equivariant map $f: P \rightarrow M$, then $\Theta^{*}\left(f^{*} \widehat{\mathcal{L}}\right) \in \operatorname{Grb}_{\nabla}\left(\Sigma^{2}\right)$. We simply define the equivariant holonomy along $(P, \Theta, f): \Sigma^{2} \rightarrow \mathcal{E}_{\nabla} G \times_{G} M$ as

$$
\operatorname{Hol}_{\widehat{\mathcal{L}}}(P, \Theta, f):=\operatorname{Hol}_{\Theta *(f * \widehat{\mathcal{L}})} \in \mathbb{R} / \mathbb{Z} .
$$

The equivariant holonomy is invariant under gauge transformations $\varphi: P \rightarrow P$, in that

$$
\operatorname{Hol}_{\widehat{\mathcal{L}}}\left(P, \varphi^{*} \Theta, f \circ \varphi\right)=\operatorname{Hol}_{\widehat{\mathcal{L}}}(P, \Theta, f) .
$$

Furthermore, if $W^{3}$ is an oriented 3-manifold, then the equivariant holonomy along the boundary of $(Q, \Theta, F): W^{3} \rightarrow \mathcal{E}_{\nabla} G \times_{G} M$ is given by the integrating the image of the equivariant curvature under the Weil homomorphism,

$$
\operatorname{Hol}_{\widehat{\mathcal{L}}}(\partial(Q, \Theta, F))=\int_{W^{3}} \operatorname{curv}\left(\Theta^{*}\left(F^{*} \widehat{\mathcal{L}}\right)\right)=\int_{W^{3}} \Theta^{*}\left(F^{*} \operatorname{curv}_{G}(\widehat{\mathcal{L}})\right) \bmod \mathbb{Z} .
$$

Therefore, one can consider the equivariant holonomy of objects in $G-G_{r b}(M)$ as a way to define WZW terms in gauged sigma models [Wit, FOS, GSW1, GSW2, BE].

Example 5.1. Consider $G=U(n), M=\mathrm{pt}$, and the topologically trivial gerbe $\mathcal{I} \in$ $U(n)-\operatorname{Grb}_{\nabla}(\mathrm{pt}) \cong \operatorname{Grb}_{\nabla}\left(\mathcal{B}_{\nabla} U(n)\right)$. Since $H^{3}(B U(n) ; \mathbb{Z})=\Omega_{U(n)}^{3}(\mathrm{pt})=0$, the short exact sequence (2.23) induces an isomorphism

$$
H^{2}(B U(n) ; \mathbb{R}) \otimes \mathbb{R} / \mathbb{Z} \stackrel{\cong}{\longrightarrow} \widehat{H}_{U(n)}^{3}(\mathrm{pt}),
$$

indicating that there can still exist non-trivial equivariant connections. To see these explicitly, consider a multiple of the universal first Chern form. For any $t \in \mathbb{R}$, define

$$
t:=t \frac{i}{2 \pi} \operatorname{Tr}(\bullet) \in\left(S^{1} \mathfrak{u}(n)^{*}\right)^{U(n)}=\Omega_{U(n)}^{2}(\mathrm{pt}) \cong H^{2}(B U(n) ; \mathbb{R}) .
$$

Then, $\left[\widehat{\mathcal{I}}_{t}\right]=0 \in \widehat{H}_{U(n)}^{3}(\mathrm{pt})$ if and only if $t \in \mathbb{Z}$. In fact, the equivariant holonomy along any 2-cycle $(P, \Theta): \Sigma^{2} \rightarrow \mathcal{B}_{\nabla} U(n)$ is

$$
\operatorname{Hol}_{\widehat{\mathcal{I}}_{t}}(P, \Theta)=\int_{\Sigma^{2}} \Theta^{*}\left(t \frac{i}{2 \pi} \operatorname{Tr}(\bullet)\right)=t \int_{\Sigma^{2}} c_{1}(\Theta) \bmod \mathbb{Z}
$$


This example illustrates why the gerbe connections in this paper should be thought of as $G$-equivariant, as opposed to merely $G$-invariant. Furthermore, it explicitly demonstrates that the category of equivariant gerbe connections considered by Gomi in [Gom1, Gom2] is not equivalent to $G-\mathrm{Grb}_{\nabla}(M)$; in particular, compare [Gom1, Proposition 4.6 and Theorem 5.16] with Theorem 4.15 and the short exact sequence $(2.22)$.

5.2. Equivariant gerbes over compact semisimple Lie groups. Let $G$ be a finitedimensional Lie group, viewed as a $G$-manifold under the adjoint action of $G$ on itself. To emphasize the action is conjugation, as opposed to left or right multiplication, we write $\mathcal{E}_{\nabla} G \times{ }_{\text {Ad }} G$ for the differential quotient stack. Note that $\left(P, \Theta, g_{\varphi}\right): X \rightarrow \mathcal{E}_{\nabla} G \times \times_{\text {Ad }} G$ is equivalent to $(P, \Theta) \in \operatorname{Bun}_{G, \nabla}(X)$, together with a gauge transformation $\varphi: P \rightarrow P$. Hence, $\widehat{\mathscr{L}} \in \operatorname{Grb}_{\nabla}\left(\mathcal{E}_{\nabla} G \times{ }_{\text {Ad }} G\right)$ associates to any such $\left(P, \Theta, g_{\varphi}\right)$ an ordinary gerbe with connection on $X$.

Gerbes on $G$ are the largest source of equivariant gerbes in the existing literature. Detailed constructions, in various forms, have been studied in [BXZ, Bry2, GR, Mei, MW]. While it is often desirable to have explicit geometric models, it is not always strictly necessary, and we now give a complimentary approach that requires very little work.

Let $\theta, \bar{\theta} \in \Omega^{1}(G ; \mathfrak{g})$ denote the left-invariant and right-invariant, respectively, MaurerCartan forms on $G$. If $G$ is compact semi-simple, then $H^{1}(G ; \mathbb{R})=H^{2}(G ; \mathbb{R})=0[\mathrm{CE}$, Theorem 21.1]. Hence $H^{3}(G ; \mathbb{R}) \cong H^{4}(B G ; \mathbb{R}) \cong\left(S^{2} \mathfrak{g}^{*}\right)^{G}$, allowing us to identify any element $\eta \in H^{3}(G ; \mathbb{R})$ with $\langle,\rangle_{\eta}$, an Ad-invariant symmetric bilinear form on $\mathfrak{g}$. We normalize so that $\eta$ is canonically represented by the closed bi-invariant 3 -form $\langle\theta,[\theta, \theta]\rangle_{\eta} \in \Omega^{3}(G)$. This 3 -form has an equivariantly closed extension [Jef, AMM]; in the Cartan model this takes the form

$$
\omega_{\eta}:=\langle\theta,[\theta, \theta]\rangle_{\eta}-\frac{1}{2}\langle\theta+\bar{\theta}, \bullet\rangle_{\eta} \in \Omega_{G}^{3}(G)
$$

where $\langle\theta+\bar{\theta}, \bullet\rangle \in\left(S^{1} \mathfrak{g}^{*} \otimes \Omega^{1}(G)\right)^{G}$. If $\eta_{G} \in H_{G}^{3}(G ; \mathbb{Z})$, we will denote its image under $H_{G}^{3}(G ; \mathbb{Z}) \rightarrow H_{G}^{3}(G ; \mathbb{R}) \cong H^{3}(G ; \mathbb{R})$ by $\eta$. The last map is an isomorphism by the following lemma.

Lemma 5.3. If $G$ is a compact semisimple Lie group, acting on itself by conjugation, then

$$
H_{G}^{2}(G ; \mathbb{R})=0 \text { and } H_{G}^{3}(G ; \mathbb{R}) \cong H^{3}(G ; \mathbb{R}) .
$$

Proof. Consider the standard Serre spectral sequence

$$
E_{2}^{i, j}=H^{i}\left(B G ; H^{j}(G ; \mathbb{R})\right) \Rightarrow H_{G}^{i+j}(G ; \mathbb{R})
$$

induced by the fibration $G \hookrightarrow E G \times{ }_{\mathrm{Ad}} G \rightarrow B G$. Since $G$ is compact semisimple, then $H^{j}(G ; \mathbb{R})=0$ for $0<j<3$, and $H^{i}(B G ; \mathbb{R})=0$ for $0<i<4$. Therefore, all terms of total degree 2 in the $E_{2}$-page vanish, and $H_{G}^{2}(G ; \mathbb{R})=0$. The only non-zero term of degree 3 is $E^{0,3}=H^{0}\left(B G ; H^{3}(G ; \mathbb{R})\right) \cong H^{3}(G ; \mathbb{R})$. The construction (5.2) of $\omega_{\eta}$ shows that every $\eta \in H^{3}(G ; \mathbb{R})$ admits an equivariant extension to $H_{G}^{3}(G ; \mathbb{R})$. Hence $E_{2}^{0,3}=E_{\infty}^{0,3}$, giving the desired isomorphism $H^{3}(G ; \mathbb{R}) \cong H_{G}^{3}(G ; \mathbb{R})$.

Theorem 5.4. Let $G$ be a compact semisimple Lie group. For any $\eta_{G} \in H_{G}^{3}(G ; \mathbb{Z})$, there exists an equivariant gerbe with equivariant connection $\widehat{\mathcal{L}}_{\eta} \in G-\mathrm{Grb}_{\nabla}(G)$ satisfying

$$
\operatorname{DD}_{G}\left(\widehat{\mathcal{L}}_{\eta}\right)=\eta_{G} \in H_{G}^{3}(G ; \mathbb{Z}), \quad \operatorname{curv}_{G}\left(\widehat{\mathcal{L}}_{\eta}\right)=\omega_{\eta} \in \Omega_{G}^{3}(G),
$$

and such an $\widehat{\mathcal{L}}_{\eta}$ is unique up to isomorphism. 
Proof. Combining Theorem 4.15, the short exact sequence (2.23)

$$
0 \rightarrow \frac{H_{G}^{2}(G ; \mathbb{R})}{H_{G}^{2}(G ; \mathbb{R})_{\mathbb{Z}}} \rightarrow \widehat{H}_{G}^{3}(M) \stackrel{\left(\mathrm{DD}_{G}, \operatorname{curv}_{G}\right)}{\longrightarrow} A_{G}^{3}(G) \rightarrow 0
$$

and the fact from Lemma 5.3 that $H_{G}^{2}(G ; \mathbb{R})=0$, we obtain isomorphisms

$$
\pi_{0}\left(G-\operatorname{Grb}_{\nabla}(G)\right) \cong \widehat{H}_{G}^{3}(G) \cong A_{G}^{3}(G) .
$$

Let $\eta_{G} \in H_{G}^{3}(G ; \mathbb{Z})$. By the definition (5.2) of $\omega_{\eta} \in \Omega_{G}^{3}(G)$ and Lemma 5.3, $\eta_{G}$ and $\omega_{\eta}$ map to the same element in $H_{G}^{3}(G ; \mathbb{R})$. In other words, $\left(\eta_{G}, \omega_{\eta}\right) \in A_{G}^{3}(G)$. Therefore, there exists a unique (up to isomorphism) $\widehat{\mathcal{L}}_{\eta} \in G-\mathrm{Grb}_{\nabla}(G)$ with the desired characteristic class and curvature.

5.3. Higher gerbes. Let $p \operatorname{Grb}_{\nabla}(M)$ denote the $(p+1)$-groupoid of $p$-gerbes with connection. The cases of $p=0$ and $p=1$ correspond to $S^{1}$-bundles and ordinary gerbes, respectively. Let

$$
\mathcal{B}_{\nabla}^{p+1} S^{1} \simeq \mathbb{L}\left(\Gamma\left(S^{1} \stackrel{d}{\rightarrow} \Omega^{1} \stackrel{d}{\rightarrow} \Omega^{2} \stackrel{d}{\rightarrow} \cdots \stackrel{d}{\rightarrow} \Omega^{p+1}\right)\right) \in \operatorname{Shv}_{\infty}
$$

denote the sheaf of $p$-groupoids that classifies $p$-gerbes with connection. This defines $\mathcal{B}_{\nabla}^{p+1} S^{1}$ up to equivalence, and specific models of $p$-gerbes yield specific constructions of $\mathcal{B}_{\nabla}^{p+1} S^{1}$.

If $M \in G$-Man, it is now reasonable to define $G$-equivariant $p$-gerbe connections on $M$ as

$$
p \operatorname{Grb}_{\nabla}\left(\mathcal{E}_{\nabla} G \times_{G} M\right):=\operatorname{Shv}_{\infty}\left(\mathcal{E}_{\nabla} G \times_{G} M, \mathcal{B}_{\nabla}^{p+1} S^{1}\right) .
$$

As seen in (2.17) and Theorem 4.16, this recovers something equivalent to the more familiar geometric notions of $G$-Bun $S^{1}, \nabla(M)$ and $G$-Grb $\operatorname{Gr}_{\nabla}(M)$ when $p=0$ and $p=1$, respectively. Furthermore, objects in $p \operatorname{Grb}_{\nabla}\left(\mathcal{E}_{\nabla} G \times_{G} M\right)$ have the same desirable formal properties as $\operatorname{Grb}_{\nabla}\left(\mathcal{E}_{\nabla} G \times_{G} M\right)$. They assign to any $(P, \Theta, f): X \rightarrow \mathcal{E}_{\nabla} G \times_{G} M$ a $p$-gerbe with connection on $X$, and to any map between principal $G$-bundles is assigned a morphism of $p$-gerbes as indicated in (4.1). Furthermore, isomorphism classes are naturally classified by the differential equivariant cohomology groups

$$
\pi_{0}\left(p-\operatorname{Grb}_{\nabla}\left(\mathcal{E}_{\nabla} G \times_{G} M\right)\right) \cong \widehat{H}_{G}^{p+2}(M)
$$

which fit into the short exact sequences (2.21)-(2.23) and a character diagram analogous to Theorem 4.15 .

\section{REFERENCES}

[AC] Camilo Arias Abad and Marius Crainic. Representations up to homotopy and Bott's spectral sequence for Lie groupoids. Adv. Math., 248:416-452, 2013.

[AMM] Anton Alekseev, Anton Malkin, and Eckhard Meinrenken. Lie group valued moment maps. $J$. Differential Geom., 48(3):445-495, 1998.

$\left[\mathrm{BCM}^{+}\right]$Peter Bouwknegt, Alan L. Carey, Varghese Mathai, Michael K. Murray, and Danny Stevenson. Twisted $K$-theory and $K$-theory of bundle gerbes. Comm. Math. Phys., 228(1):17-45, 2002.

[BE] Daniel Berwick-Evans. Twisted equivariant elliptic cohomology from gauged perturbative sigma models I: Finite gauge groups, 2014. arXiv:1410.5500.

[BNV] Ulrich Bunke, Thomas Nikolaus, and Michael Völkl. Differential cohomology theories as sheaves of spectra. J. Homotopy Relat. Struct., 11(1):1-66, 2016.

[Bro] Kenneth S. Brown. Abstract homotopy theory and generalized sheaf cohomology. Trans. Amer. Math. Soc., 186:419-458, 1973.

[Bry1] Jean-Luc Brylinski. Loop spaces, characteristic classes and geometric quantization, volume 107 of Progress in Mathematics. Birkhäuser Boston Inc., Boston, MA, 1993.

[Bry2] Jean-Luc Brylinski. Gerbes on complex reductive Lie groups, 2000. arXiv:math/0002158. 
[BSS] Marco Benini, Alexander Schenkel, and Richard J. Szabo. Homotopy colimits and global observables in abelian gauge theory. Lett. Math. Phys., 105(9):1193-1222, 2015.

[BTW] Ulrich Bunke, Paul Turner, and Simon Willerton. Gerbes and homotopy quantum field theories. Algebr. Geom. Topol., 4:407-437, 2004.

[BV] Nicole Berline and Michèle Vergne. Zéros d'un champ de vecteurs et classes caractéristiques équivariantes. Duke Math. J., 50(2):539-549, 1983.

[BXZ] Kai Behrend, Ping Xu, and Bin Zhang. Equivariant gerbes over compact simple Lie groups. C. $R$. Math. Acad. Sci. Paris, 336(3):251-256, 2003.

[CE] Claude Chevalley and Samuel Eilenberg. Cohomology theory of Lie groups and Lie algebras. Trans. Amer. Math. Soc., 63:85-124, 1948.

[CS] Jeff Cheeger and James Simons. Differential characters and geometric invariants. In Geometry and topology (College Park, Md., 1983/84), volume 1167 of Lecture Notes in Math., pages 50-80. Springer, Berlin, 1985.

[Del] Pierre Deligne. Théorie de Hodge. II. Inst. Hautes Études Sci. Publ. Math., (40):5-57, 1971.

[Dug] Daniel Dugger. A primer on homotopy colimits. http://math.uoregon.edu/ ddugger/hocolim.pdf.

[FH] Daniel S. Freed and Michael J. Hopkins. Chern-Weil forms and abstract homotopy theory. Bull. Amer. Math. Soc. (N.S.), 50(3):431-468, 2013.

[FNSW] Jürgen Fuchs, Thomas Nikolaus, Christoph Schweigert, and Konrad Waldorf. Bundle gerbes and surface holonomy. In European Congress of Mathematics, pages 167-195. Eur. Math. Soc., Zürich, 2010 .

[FOS] José M. Figueroa-O'Farrill and Sonia Stanciu. Gauged Wess-Zumino terms and equivariant cohomology. Phys. Lett. B, 341(2):153-159, 1994.

[Fre] Daniel S. Freed. On equivariant Chern-Weil forms and determinant lines, 2016. arXiv:1606.01129.

[FSS] Domenico Fiorenza, Urs Schreiber, and Jim Stasheff. Čech cocycles for differential characteristic classes: an $\infty$-Lie theoretic construction. Adv. Theor. Math. Phys., 16(1):149-250, 2012.

[Gaw] Krzysztof Gawedzki. Bundle gerbes for topological insulators, 2015. arXiv:1512.01028.

[GL] Alexander Gorokhovsky and John Lott. A Hilbert bundle description of differential K-theory, 2015. arXiv:1512.07185.

[Gom1] Kiyonori Gomi. Equivariant smooth Deligne cohomology. Osaka J. Math., 42(2):309-337, 2005.

[Gom2] Kiyonori Gomi. Relationship between equivariant gerbes and gerbes over the quotient space. Commun. Contemp. Math., 7(2):207-226, 2005.

[GR] Krzysztof Gawedzki and Nuno Reis. WZW branes and gerbes. Rev. Math. Phys., 14(12):1281-1334, 2002.

[GS] Victor W. Guillemin and Shlomo Sternberg. Supersymmetry and equivariant de Rham theory. Mathematics Past and Present. Springer-Verlag, Berlin, 1999.

[GSW1] Krzysztof Gawędzki, Rafał R. Suszek, and Konrad Waldorf. Global gauge anomalies in twodimensional bosonic sigma models. Comm. Math. Phys., 302(2):513-580, 2011.

[GSW2] Krzysztof Gawędzki, Rafał R. Suszek, and Konrad Waldorf. The gauging of two-dimensional bosonic sigma models on world-sheets with defects. Rev. Math. Phys., 25(6):1350010, 122, 2013.

[HQ] Michael J. Hopkins and Gereon Quick. Hodge filtered complex bordism. J. Topol., 8(1):147-183, 2015.

[Jef] Lisa C. Jeffrey. Group cohomology construction of the cohomology of moduli spaces of flat connections on 2-manifolds. Duke Math. J., 77(2):407-429, 1995.

[Kal] Jaap Kalkman. BRST model for equivariant cohomology and representatives for the equivariant Thom class. Comm. Math. Phys., 153(3):447-463, 1993.

[KT] Andreas Kübel and Andreas Thom. Equivariant Differential Cohomology, 2015. arXiv:1510.06392.

[KV] Alexander Kahle and Alessandro Valentino. T-duality and differential K-theory. Commun. Contemp. Math., 16(2):1350014, 27, 2014.

[Lur] Jacob Lurie. Higher topos theory, volume 170 of Annals of Mathematics Studies. Princeton University Press, Princeton, NJ, 2009.

[Mei] Eckhard Meinrenken. The basic gerbe over a compact simple Lie group. Enseign. Math. (2), 49(34):307-333, 2003.

[MP] Marco Mackaay and Roger Picken. Holonomy and parallel transport for abelian gerbes. Adv. Math., 170(2):287-339, 2002.

[MQ] Varghese Mathai and Daniel Quillen. Superconnections, Thom classes, and equivariant differential forms. Topology, 25(1):85-110, 1986. 
[MRSV] Michael K. Murray, David Michael Roberts, Danny Stevenson, and Raymond F. Vozzo. Equivariant bundle gerbes. Adv. Theor. Math. Phys., 21(4), 2017. arXiv:1506.07931.

[MT] Varghese Mathai and Guo Chuan Thiang. Differential Topology of Semimetals. Comm. Math. Phys., 355(2):561-602, 2017.

[Mur] M. K. Murray. Bundle gerbes. J. London Math. Soc. (2), 54(2):403-416, 1996.

[MW] Jouko Mickelsson and Stefan Wagner. Third group cohomology and gerbes over Lie groups. J. Geom. Phys., 108:49-70, 2016.

[NS] Thomas Nikolaus and Christoph Schweigert. Equivariance in higher geometry. Adv. Math., 226(4):3367-3408, 2011.

[Par] Byungdo Park. Geometric models of twisted differential K-theory I. J. Homotopy Relat. Struct., 2017. arXiv:1602.02292.

[Red1] Corbett Redden. Differential Borel equivariant cohomology via connections. New York J. Math., 23:441-487, 2017.

[Red2] Corbett Redden. An alternate description of equivariant connections, 2016. arXiv:1608.01297.

[SSW] Urs Schreiber, Christoph Schweigert, and Konrad Waldorf. Unoriented WZW models and holonomy of bundle gerbes. Comm. Math. Phys., 274(1):31-64, 2007.

[Ste] Danny Stevenson. The Geometry of Bundle Gerbes. PhD thesis, University of Adelaide, 2000. arXiv:math.DG/0004117.

[Sti] Mathieu Stiénon. Equivariant Dixmier-Douady classes. Math. Res. Lett., 17(1):127-145, 2010.

[TX] Jean-Louis Tu and Ping Xu. Periodic Cyclic Homology and Equivariant Gerbes, 2015. arXiv:1504.08064.

[Wal1] Konrad Waldorf. More morphisms between bundle gerbes. Theory Appl. Categ., 18:No. 9, 240-273, 2007.

[Wal2] Konrad Waldorf. String connections and Chern-Simons theory. Trans. Amer. Math. Soc., 365(8):4393-4432, 2013.

[Wit] Edward Witten. On holomorphic factorization of WZW and coset models. Comm. Math. Phys., 144(1):189-212, 1992.

Hausdorff Research Institute for Mathematics (HIM), Poppelsdorfer Allee 45, 53115 Bonn, Germany

Current address: School of Mathematics, Korea Institute for Advanced Study, 85 Hoegiro, Dongdaemungu, Seoul, 02455, Republic of Korea

E-mail address: byungdpark@gmail.com

Department of Mathematics, LiU Post, Long Island University, 720 Northern Blvd, Brookville, NY 11548, USA

E-mail address: corbett.redden@liu.edu 